\title{
Reliable Computation of Equilibrium States and Bifurcations in Food Chain Models
}

\author{
C. Ryan Gwaltney, Mark P. Styczynski ${ }^{\dagger}$, and Mark A. Stadtherr ${ }^{*}$ \\ Department of Chemical and Biomolecular Engineering \\ University of Notre Dame \\ 182 Fitzpatrick Hall \\ Notre Dame, IN 46556 \\ USA
}

${ }^{\dagger}$ Current Address: Department of Chemical Engineering, Massachusetts Institute of Technology, Building 66, 25 Ames Street, Cambridge MA 02139 USA

*Author to whom correspondence should be addressed: Tel.: (574) 631-9318, Fax: (574) 631-8366, E-mail: markst@nd.edu

(July 2003)

(revised, March 2004) 


\begin{abstract}
Food chains and webs in the environment can be modeled by systems of ordinary differential equations that approximate species or functional feeding group behavior with a variety of functional responses. We present here a new methodology for computing all equilibrium states and bifurcations of equilibria in food chain models. The methodology used is based on interval analysis, in particular an interval-Newton/generalized-bisection algorithm that provides a mathematical and computational guarantee that all roots of a nonlinear equation system are enclosed. The procedure is initialization-independent, and thus requires no a priori insights concerning the number of equilibrium states and bifurcations of equilibria or their approximate locations. The technique is tested using several example problems involving tritrophic food chains.
\end{abstract}

Keywords: Food chain; Ecology, Nonlinear dynamics; Bifurcations; Interval analysis 


\section{INTRODUCTION}

Food chains and webs in the environment are highly complex and interdependent systems. Seemingly insignificant changes in the parameters of such systems can have drastic consequences. Food chains and webs can be modeled by systems of ordinary differential equations that approximate species or functional feeding group behavior with a variety of functional responses. Many simple two-species models have been thoroughly explored, while new discoveries continue to be made in examining models with three and four trophic levels (e.g., Moghadas and Gumel, 2003). Ecological systems exhibit complex interdependencies in that changes in a single trophic level may have far reaching impacts on the rest of the system. In some cases, this leads to unexpected or counterintuitive behavior. Use of simple food chain models can assist in qualitatively illustrating the complexity and interdependencies in real ecological systems.

Our interest in ecological modeling is motivated by its use as one tool in studying the impact on the environment of the industrial use of newly discovered materials. Clearly it is preferable to take a proactive, rather than reactive, approach when considering the safety and environmental consequences of using new compounds. Of particular interest is the potential use of room temperature ionic liquid (IL) solvents in place of traditional solvents (Brennecke and Maginn, 2001). IL solvents have no measurable vapor pressure and thus, from a safety and environmental viewpoint, have several potential advantages relative to the traditional volatile organic compounds (VOCs) used as solvents, including elimination of hazards due to inhalation, explosion and air pollution. However, ILs are, to varying degrees, soluble in water; thus, if they are used industrially on a large scale, their entry into the environment via aqueous waste streams is of concern. The effects of trace levels of ILs in the environment are today essentially unknown and thus must be studied. Single species toxicity information is very important as a basis for examining the effects that a contaminant will have on an environment. However, this information, when considered by itself, is insufficient to predict impacts on a food chain, food web, or an ecosystem. Ecological modeling provides a means for studying the impact of such perturbations on a localized environment by focusing not just on the impact on one species, but rather on the larger impacts on the food chain and ecosystem. Of course, ecological modeling is just one part of a much larger suite of tools, including toxicological (Jastorff et al., 2003; 
Freemantle, 2002), hydrological and microbiological studies, that must be used in addressing this issue.

In this paper, we concentrate on the computation of equilibrium states (steady states) in food chain models, and on the computation of bifurcations of equilibria. A bifurcation is a sudden, macroscopic change in the qualitative behavior of a system as some parameter is varied. These changes include the appearance and disappearance of equilibrium states (fold or saddle node bifurcation), the exchange of stability of two equilibria (transcritical bifurcation), and the change of stability of an equilibrium point (Hopf bifurcation). van Coller (1997) provides a good high-level introduction for dynamical systems and their characteristics, while a more advanced and thorough review of bifurcations can be found in Kuznetsov (1998). For simple systems, or specific parts of more complex ones, analytic techniques and isocline analysis are useful for analysis of equilibrium states and bifurcations. However, for more complex problems, continuation methods are the predominant computational tools, with packages such as AUTO (Doedel et al., 1997) and others (van Coller, 1997) being particularly popular in this context. These are applied to solve the systems of nonlinear algebraic equations that represent the equilibrium states and bifurcations.

Continuation methods can be quite reliable, especially in the hands of an experienced user. However, in general, continuation methods are initialization dependent, and may fail to find all solutions to a system of nonlinear equations. Thus, in this context, these methods may fail to find all equilibrium states or all bifurcations of equilibria. In this paper, we propose a new approach for computing equilibrium states and bifurcations of equilibria in food chain models, and consider the feasibility of using this approach. This technique is based on interval mathematics, in particular an interval-Newton approach combined with generalized bisection, and provides a mathematical and computational guarantee that all equilibrium states and bifurcations of equilibria will be located (or, more precisely, enclosed within a very narrow interval). There are other dynamical features of interest, such as limit cycles (and their bifurcations); however, our attention here will be limited to equilibrium states and their bifurcations. While the focus here is on food chain models, there are clearly applications of this technique in the analysis of other dynamical systems of interest in chemical engineering.

In the next section, we describe the development of food chain models and the formulation of the nonlinear equation systems that must be solved to determine equilibrium 
states and bifurcations. In Section 3, the computational method to be considered is described briefly. Then, in Section 4, we apply this methodology to some relatively simple systems to explore its feasibility.

\section{PROBLEM FORMULATION}

\subsection{Food Chain Models}

The food chain models studied in this paper are all continuous time models that are represented by a set of ordinary differential equations. These expressions give the rate of change of biomass in terms of specific models of growth and mortality at each trophic level. In food chain models, it is common to equate each trophic level with a single species, and that is the practice that we will follow here. However, it should be noted that a trophic level may in fact consist of multiple similar (and noncompetitive) species with the same functional feeding behavior. Species biomass can be related to species population by considering the average size and mass of individual members of a species. However, it is convenient to work in terms of biomass for many organisms, especially those found in aquatic food chains. Thus, when the term population is used here, it refers to species biomass.

In general, for a food chain with $N$ trophic levels, the equations giving the rate of change of biomass for each trophic level $i$ (species $i$ ) can be expressed as:

$$
\frac{d x_{i}}{d t}=g_{i}-m_{i}, \quad i=1, \ldots, N
$$

where $x_{i}$ is the species biomass, $g_{i}$ is the species growth rate, and $m_{i}$ is the species removal (mortality) rate. The removal rate of a species may include deaths due to natural causes, predation, harvesting, contamination, etc., and also includes the net number of individuals leaving the control volume of interest, whether due to drift or washout. The species growth rate may include growth due to consumption of prey, or due to consumption of nutrients.

At the lowest level of the food chain (species 1), simple prey species are typically modeled as growing either exponentially or logistically in the absence of a predator. Logistic models tend to better represent real systems, as these models account for the effect of prey density on growth. The logistic growth model is: 


$$
g_{1}=x_{1}\left[r\left(1-\frac{x_{1}}{K}\right)\right]
$$

where $r$ is the prey growth rate constant and $K$ is the prey carrying capacity for the system. In this type of model, at small prey populations, prey species grow exponentially. However, as the population gets larger, the rate of growth slows until the population reaches the system carrying capacity. This carrying capacity represents the maximum biomass of a prey species that a system can support when the prey lives in absence of a predator. This growth rate model represents the net growth, including both birth and natural death, of prey organisms. This type of model is sufficient to characterize population dynamics of simple organisms at the bottom of a food chain. Predation behavior is quite different, however.

Predators (species $2, \ldots, N$ ) grow by consuming prey, and the rate at which predators consume prey can modeled by different response types. Holling (1959) categorized predator responses into three classes, as explained in more detail by Turchin (2003). Type I predators exhibit a linear functional response, while Type II and Type III predators exhibit hyperbolic and sigmoidal responses, respectively. The corresponding growth rate models are:

Linear: $\quad g_{i}=e_{i} a_{i} x_{i} x_{i-1}$

Hyperbolic: $\quad g_{i}=e_{i} \frac{a_{i} x_{i} x_{i-1}}{b_{i}+x_{i-1}}$

Sigmoidal: $\quad g_{i}=e_{i} \frac{a_{i} x_{i} x_{i-1}^{2}}{b_{i}^{2}+x_{i-1}^{2}}$.

Here $x_{i}$ is the predator species biomass, $x_{i-1}$ is the prey species biomass, $a_{i}$ is the maximum predation rate, $b_{i}$ is a half-saturation constant, and $e_{i}$ is the efficiency with which a predator converts prey into biomass. If the efficiency $e_{i}$ equals one, then $g_{i}$ represents the rate of prey consumption. Note that in a food chain model (as opposed to a food web), a predator species preys only on the trophic level immediately below it in the chain. Because of the amount of time required to handle prey (i.e., hunt, kill, consume, digest), predator species cannot consume prey at a constant rate as prey population increases. Thus, linear functional responses do not accurately portray behavior in the natural environment. On the other hand, the hyperbolic and sigmoidal functional responses provide a saturation effect based upon the prey species population. One important difference between the hyperbolic response and the sigmoidal 
response arises as the prey population diminishes towards zero. As the prey population approaches zero, the rate of change in prey consumption rate modeled by the hyperbolic response increases, while the rate of change in prey consumption rate modeled by the sigmoidal model response passes through an inflection point, and then decreases. This means that as prey population dwindles to a very low level, predators exhibiting a sigmoidal response slow in their efforts to consume prey, while hyperbolic predators work harder for their meals. This reduction in effort by sigmoidal predators to catch prey is typical of a generalist predator that switches to another food source when prey abundance becomes low. The hyperbolic response is characteristic of specialist predators, which do not alternate food sources. Specialist predation is generally seen as a more accurate representation of many systems, including aquatic systems; however, both types of behavior can be used to model natural systems.

The species removal rate generally involves two terms, one accounting for death by predation, and the other being a density-dependant death rate term accounting for natural death and other forms of removal (e.g., harvesting, washout, etc.). The loss of biomass by predation at one trophic level is directly related to the growth by predation at the next highest level in the food chain, and differs only by the efficiency factor introduced above. Thus, for example, the removal rate for a species $i$ with a hyperbolic predator (species $i+1$ ) would be represented by:

$$
m_{i}=\frac{a_{i+1} x_{i+1} x_{i}}{b_{i+1}+x_{i}}+d_{i} x_{i}
$$

where $d_{i}$ is the death rate constant. Note that the form of the first term (removal by predation) depends on the form of the predator growth rate. Expressions such as this for $m_{i}$, which include both the predation term and the density-dependent death rate term, will apply for $i=2, \ldots, N-1$. For the bottom prey species $(i=1)$, there is no density-dependent death rate term as this is accounted for in the logistic growth rate model. For the top predator species $(i=N)$, there is no consumption by predation term, since there is no predator higher in the food chain.

Based on the concepts outlined above, one can form a model of a food chain consisting of any number of species that exhibit a variety of functional responses. For example, consider a tritrophic $(N=3)$ chain with a logistic prey $(i=1)$, and hyperbolic (Holling Type II) predator $(i=$ $2)$ and superpredator $(i=3)$ responses: 


$$
\begin{aligned}
& \frac{d x_{1}}{d t}=x_{1} r\left(1-\frac{x_{1}}{K}\right)-\frac{a_{2} x_{1} x_{2}}{b_{2}+x_{1}}=x_{1}\left[r\left(1-\frac{x_{1}}{K}\right)-\frac{a_{2} x_{2}}{b_{2}+x_{1}}\right] \\
& \frac{d x_{2}}{d t}=e_{2} \frac{a_{2} x_{1} x_{2}}{b_{2}+x_{1}}-\frac{a_{3} x_{2} x_{3}}{b_{3}+x_{2}}-d_{2} x_{2}=x_{2}\left[e_{2} \frac{a_{2} x_{1}}{b_{2}+x_{1}}-\frac{a_{3} x_{3}}{b_{3}+x_{2}}-d_{2}\right] \\
& \frac{d x_{3}}{d t}=e_{3} \frac{a_{3} x_{2} x_{3}}{b_{3}+x_{2}}-d_{3} x_{3}=x_{3}\left[e_{3} \frac{a_{3} x_{2}}{b_{3}+x_{2}}-d_{3}\right]
\end{aligned}
$$

This model is well-known as a tritrophic Rosenzweig-MacArthur model (also referred to as a tritrophic Oksanen model), and is frequently used in theoretical ecology (e.g., Gragnani et al., 1998; Hastings and Powell, 1991; Klebanoff and Hastings, 1994; Abrams and Roth, 1994; Kuznetsov and Rinaldi, 1996; De Feo and Rinaldi, 1997). Since this model is relatively simple and has been widely studied both analytically and numerically, it provides a good initial problem for testing the feasibility of the interval-based methodology described below for determining equilibrium states and bifurcations of equilibria in food chain models.

Two additional tritrophic models, involving different predator functional responses, will be used as test problems. The first of these involves a sigmoidal (Holling Type III) predator and superpredator, and is given by:

$$
\begin{aligned}
& \frac{d x_{1}}{d t}=x_{1}\left[r\left(1-\frac{x_{1}}{K}\right)-\frac{a_{2} x_{1} x_{2}}{b_{2}^{2}+x_{1}^{2}}\right] \\
& \frac{d x_{2}}{d t}=x_{2}\left[e_{2} \frac{a_{2} x_{1}^{2}}{b_{2}^{2}+x_{1}^{2}}-\frac{a_{3} x_{2} x_{3}}{b_{3}^{2}+x_{2}^{2}}-d_{2}\right] \\
& \frac{d x_{3}}{d t}=x_{3}\left[e_{3} \frac{a_{3} x_{2}^{2}}{b_{3}^{2}+x_{2}^{2}}-d_{3}\right]
\end{aligned}
$$

This model appears to have received only limited study (Turchin, 2003; Yodzis, 1989), as the Type III functional response is generally only applicable to generalist, not specialist, predators, and is thus perhaps less widely applicable in typical natural environments than the RosenzweigMacArthur model.

The second of the two additional test problems involves a hyperbolic, or specialist, predator and a sigmoidal, or generalist, superpredator. This model is given by: 


$$
\begin{aligned}
& \frac{d x_{1}}{d t}=x_{1}\left[r\left(1-\frac{x_{1}}{K}\right)-\frac{a_{2} x_{2}}{b_{2}+x_{1}}\right] \\
& \frac{d x_{2}}{d t}=x_{2}\left[e_{2} \frac{a_{2} x_{1}}{b_{2}+x_{1}}-\frac{a_{3} x_{2} x_{3}}{b_{3}^{2}+x_{2}^{2}}-d_{2}\right] \\
& \frac{d x_{3}}{d t}=x_{3}\left[e_{3} \frac{a_{3} x_{2}^{2}}{b_{3}^{2}+x_{2}^{2}}-d_{3}\right]
\end{aligned}
$$

This model has apparently not been widely studied.

Note that, since all of the terms typically used to model $g_{i}$ and $m_{i}$ have a common factor of $x_{i}$, food chain models, including all of the models above, can typically be represented by the general form

$$
\frac{d \boldsymbol{x}}{d t}=\boldsymbol{x}^{\mathrm{T}} \boldsymbol{h}(\boldsymbol{x})
$$

where $\boldsymbol{x}=\left[x_{1}, x_{2}, \ldots, x_{N}\right]^{\mathrm{T}}$ is the vector of population (biomass) densities, and $\boldsymbol{h}=\left[h_{1}, h_{2}, \ldots, h_{N}\right]^{\mathrm{T}}$ is the vector of functions $h_{i}(\boldsymbol{x})=\left[g_{i}(\boldsymbol{x})-m_{i}(\boldsymbol{x})\right] / x_{i}$.

In real systems, the simple food chains discussed above are likely imbedded within larger and more complex food webs, but for the purposes of many theoretical ecology studies, the tritrophic food chain has proven useful in analyzing both qualitative and quantitative trends in population fluctuation.

\subsection{Equilibrium States}

Equilibrium states are defined by the condition

$$
\frac{d \boldsymbol{x}}{d t}=\boldsymbol{x}^{\mathrm{T}} \boldsymbol{h}(\boldsymbol{x})=\mathbf{0}
$$

subject to

$$
\boldsymbol{x} \geq \mathbf{0}
$$

Once values for all of the model parameters have been specified, Eq. (17) represents an $N \times N$ system of nonlinear equations which can be solved for the equilibrium states. For food chain models as described above, there may be a large number of solutions; however, typically some of these solutions will not satisfy Eq. (18) and thus will be infeasible. Note that the solution of Eq. 
(17) can be thought of as consisting of $2^{N}$ subproblems, one for the case of all nonzero $x_{i}$ and requiring the solution of $\boldsymbol{h}(\boldsymbol{x})=\mathbf{0}$, and $2^{N}-1$ subproblems corresponding to different combinations of $x_{i}$ set to zero, each combination requiring the solution of a system $h_{i}=0, i \in S$, where $S$ indicates the set of indices corresponding to nonzero $x_{i}$. In general, each of the subproblems that must be solved (except for the case $\boldsymbol{x}=\mathbf{0}$ ) may have multiple solutions or no solutions, and so the total number of equilibrium states may be unknown a priori. For simple models, it may be possible to solve for many of the equilibrium states analytically, but for more complex models a computational method is needed that is capable of finding, with certainty, all the solutions of a nonlinear equation system. The interval-Newton procedure described below is tested here for this purpose. It is applied directly to the solution of Eq. (17) rather than to any of the several subproblems.

The stability of an equilibrium state can be determined by evaluating the Jacobian matrix at this state and then examining its eigenvalues. From Eq. (17), the Jacobian matrix $J$ of interest has the elements

$$
J_{i k}=\frac{\partial\left(x_{i} h_{i}\right)}{\partial x_{k}} .
$$

According to linear stability analysis, for an equilibrium state to be stable, all of the eigenvalues of the Jacobian must have negative real parts.

Examining equilibrium states can give us information on how the behavior of the system changes with changes in the model parameters. Since the parameters in the model are representative of physical and biological characteristics of the system, the model parameters can be altered in order to represent changes in a real system. Tracking the changes in the equilibrium states can give us information on how a real system might behave when undergoing perturbations in the system parameters.

\subsection{Codimension-One Bifurcations}

To find bifurcations of codimension one, all model parameters but one are specified, and then the values of the remaining parameter at which there is a sudden change in the nature of an equilibrium state are found. Of interest here are fold and transcritical bifurcations of equilibria and Hopf bifurcations. Mathematically, when an equilibrium state undergoes either a fold or a transcritical bifurcation, an eigenvalue of its Jacobian becomes zero. In this case, there are two 
equilibria that "collide" as the free parameter is varied. In a fold bifurcation, these equilibria mutually annihilate, thus the number of equilibrium states changes by two as the free parameter is increased or decreased. On the other hand, in a transcritical bifurcation, the two colliding equilibria do not disappear, but may simply exchange stability. In a system with a single state variable, there will always be an exchange of stability, but if the number of state variables is more than one, there may or may not be an exchange of stability, depending on the sign of the other eigenvalues. Mathematically, a Hopf bifurcation occurs when its Jacobian has a pair of complex conjugate eigenvalues and the sign of their real part changes; i.e. when this complex conjugate pair of eigenvalues is purely imaginary. In a system with two state variables, this will result in a change in the stability of the equilibrium state, but if the number of state variables is more than two, there may or may not be stability change, depending on the sign of the other eigenvalues. If the Hopf bifurcation occurs in an independent two-variable subset of state space, this is referred to as a planar Hopf bifurcation.

The locations of these bifurcations can be computed by solving a nonlinear equation system that includes the equilibrium conditions, Eq. (17), together with an augmenting (or test) function that represents the mathematical condition for the type of bifurcation sought. Kuznetsov (1998) discusses in detail the development of such test functions for the types of bifurcations of interest here. Generally these test functions are designed to avoid the need for direct computation of eigenvalues.

At a fold or transcritical bifurcation, an eigenvalue of $J$ is zero. Since the determinant of a matrix is equal to the product of its eigenvalues, the determinant of $J$ will be zero at a fold or transcritical bifurcation, thereby providing a convenient test function. Thus, to locate a fold or transcritical bifurcation of equilibrium, a nonlinear equation system that can be solved is

$$
\begin{gathered}
\boldsymbol{x}^{\mathrm{T}} \boldsymbol{h}(\boldsymbol{x}, \alpha)=\mathbf{0} \\
\operatorname{det}[J(\boldsymbol{x}, \alpha)]=0
\end{gathered}
$$

This is a system of $N+1$ equations in the $N+1$ variables $\boldsymbol{x}$ and $\alpha$, where $\alpha$ is the free model parameter.

At a Hopf bifurcation, $J$ has a complex conjugate pair of purely imaginary eigenvalues. This means that there must be a pair of eigenvalues that sums to zero (but note that the converse is not true-having a pair of eigenvalues that sums to zero does not necessarily mean that they 
are a complex conjugate pair with zero real part). According to Stephanos's theorem, for an $N \times$ $N$ matrix $J$ with eigenvalues $\lambda_{1}, \lambda_{2}, \ldots, \lambda_{N}$, the bialternate product $J \otimes J$ has eigenvalues $\lambda_{i} \lambda_{j}$ and the bialternate product $2 J \otimes I$ has eigenvalues $\lambda_{i}+\lambda_{j}$. Thus, to locate a Hopf bifurcation a nonlinear equation system that can be solved is

$$
\begin{aligned}
& \boldsymbol{x}^{\mathrm{T}} \boldsymbol{h}(\boldsymbol{x}, \alpha)=\mathbf{0} \\
& \operatorname{det}[2 J(\boldsymbol{x}, \alpha) \otimes I]=0 .
\end{aligned}
$$

Again, this is a system of $N+1$ equations in the $N+1$ variables $\boldsymbol{x}$ and $\alpha$. The bialternate product of two $n \times n$ matrices $A$ and $B$ is an $m \times m$ matrix denoted by $A \otimes B$ whose rows are labeled by the multiindex $(p, q)$ where $p=2,3, \ldots, n$ and $q=1,2, \ldots, p-1$, whose columns are labeled by the multiindex $(r, s)$ where $r=2,3, \ldots, n$ and $s=1,2, \ldots, r-1$, where $m=n(n-1) / 2$, and whose elements are given by

$$
(A \otimes B)_{(p, q)(r, s)}=\frac{1}{2}\left(\left|\begin{array}{ll}
a_{p r} & a_{p s} \\
b_{q r} & b_{q s}
\end{array}\right|+\left|\begin{array}{ll}
b_{p r} & b_{p s} \\
a_{q r} & a_{q s}
\end{array}\right|\right) .
$$

Note that solutions to this nonlinear equation system will include all Hopf bifurcations, but that there may be other solutions corresponding to the case of two eigenvalues that are real additive inverses (and for which there thus is also a pair of eigenvalues that sums to zero). To identify such "false positives" it is thus necessary to compute the eigenvalues of $J$ at each solution to Eqs. (22-23). Then any solutions for which the eigenvalues do not include a purely imaginary complex conjugate pair can be discarded.

\subsection{Codimension-Two Bifurcations}

To find bifurcations of codimension two, all but two model parameters are specified, and then the values of the two free parameters at which there is a sudden change in the nature of an equilibrium state are found, much in the same way that codimension-one bifurcations are found. On a bifurcation diagram (see examples below), codimension-two bifurcation points may occur at the intersections of codimension-one bifurcation curves; thus, these codimension-two bifurcations are of interest since they serve as "organizing centers" for the diagram. Furthermore, codimension-one bifurcation curves can exhibit qualitative, macroscopic changes in number and/or type when passing through codimension-two bifurcation points. Knowledge of the codimension-two bifurcations alone, without determination of an entire bifurcation diagram, 
can be useful for comparison of models (Gragnani et al., 1998).

Corresponding to the types of codimension-one bifurcations considered here, there are three basic types of codimension-two bifurcations. They can be classified mathematically by examining the eigenvalues of the unaugmented Jacobian $J$ defined by Eq. (19). The Jacobian can either have a pair of purely zero eigenvalues (double-fold or double-zero bifurcation), two pairs of purely imaginary complex conjugate eigenvalues (double-Hopf bifurcation), or a pair of purely imaginary complex conjugate eigenvalues and one zero eigenvalue (fold-Hopf bifurcation). Since the examples used in this paper are tritrophic, the double-Hopf case will not be considered here, as these cannot occur in a model with less than four equations (the doubleHopf condition involves four eigenvalues). There are also other types of codimension-two bifurcations (e.g., cusp bifurcation) that are not searched for directly here, but which may be encountered (see Section 4.2).

Both double-fold and fold-Hopf bifurcations can be found be solving the doubly augmented nonlinear system

$$
\begin{aligned}
& \boldsymbol{x}^{\mathrm{T}} \boldsymbol{h}(\boldsymbol{x}, \alpha, \beta)=\mathbf{0} \\
& \operatorname{det}[J(\boldsymbol{x}, \alpha, \beta)]=0 \\
& \operatorname{det}[2 J(\boldsymbol{x}, \alpha, \beta) \otimes I]=0 .
\end{aligned}
$$

This is a system of $N+2$ equations in the $N+2$ variables $\boldsymbol{x}, \alpha$, and $\beta$, where $\alpha$ and $\beta$ are the free model parameters. Eq. (26) applies since, at either a double-fold or fold-Hopf bifurcation, $J$ must have an eigenvalue of zero. Eq. (27) applies since, whether it is the pair of zero eigenvalues at a double-fold bifurcation or the pair of purely imaginary complex conjugate eigenvalues at a fold-Hopf bifurcation, $J$ must have a pair of eigenvalues that sums to zero. Once found, solutions to Eqs. (25-27) must be screened for points that have a pair of (nonzero) eigenvalues that are purely real additive inverses, and the points must be further sorted and classified by type. Whether one is looking for fold and transcritical bifurcations and solving Eqs. (20-21), looking for Hopf bifurcations and solving Eqs. (22-23), or looking for codimension-two bifurcations by solving Eqs. (25-27), the equation system that must be solved may have multiple solutions, or no solutions, and the number of solutions may be unknown a priori. A computational method is needed that is capable of finding, with certainty, all the solutions of 
these nonlinear equation systems. The interval-Newton procedure described below is tested here for this purpose.

\section{COMPUTATIONAL METHODOLOGY}

Recent monographs that introduce interval mathematics, as well as computations with intervals, include those of Neumaier (1990), Hansen (1992) and Kearfott (1996). Of particular interest here is the use of an interval-Newton/generalized-bisection (IN/GB) technique. Properly implemented, this technique provides the power to find, with mathematical and computational certainty, narrow enclosures of all solutions of a system of nonlinear equations, or to determine with certainty that there are none, provided that initial upper and lower bounds are available for all variables (Neumaier, 1990; Hansen, 1992, Kearfott, 1996). This is made possible through the use of the powerful existence and uniqueness test provided by the interval-Newton method. The key ideas of the methodology used are summarized briefly here.

Consider an $n \times n$ nonlinear equation system $\boldsymbol{f}(\boldsymbol{x})=\mathbf{0}$ with a finite number of real roots in some initial interval $\boldsymbol{X}^{(0)}$. The interval Newton methodology is applied to a sequence of subintervals of $\boldsymbol{X}^{(0)}$. For a subinterval $\boldsymbol{X}^{(k)}$ in the sequence, the first step is the function range test. An interval extension $\boldsymbol{F}\left(\boldsymbol{X}^{(k)}\right)$ of the function $\boldsymbol{f}(\boldsymbol{x})$ is calculated, which provides upper and lower bounds on the range of values of $\boldsymbol{f}(\boldsymbol{x})$ in $\boldsymbol{X}^{(k)}$. Interval extensions are computed here by substituting the given interval into the function and then evaluating the function using interval arithmetic. If there is any component of the interval extension $\boldsymbol{F}\left(\boldsymbol{X}^{(k)}\right)$ that does not include zero, then the interval can be discarded, since no solution of $\boldsymbol{f}(\boldsymbol{x})=\mathbf{0}$ can exist in this interval. The next subinterval in the sequence may then be considered. Otherwise, testing of $\boldsymbol{X}^{(k)}$ continues.

The next step is the interval-Newton test. The linear interval equation system

$$
F^{\prime}\left(\boldsymbol{X}^{(k)}\right)\left(\boldsymbol{N}^{(k)}-\boldsymbol{x}^{(k)}\right)=-\boldsymbol{f}\left(\boldsymbol{x}^{(k)}\right)
$$

is solved for a new interval $\boldsymbol{N}^{(k)}$, where $F^{\prime}\left(\boldsymbol{X}^{(k)}\right)$ is an interval extension of the Jacobian of $\boldsymbol{f}(\boldsymbol{x})$, and $\boldsymbol{x}^{(k)}$ is an arbitrary point in $\boldsymbol{X}^{(k)}$. It can be shown (Moore, 1966) that any root contained in $\boldsymbol{X}^{(k)}$ is also contained in the image $\boldsymbol{N}^{(k)}$. This implies that when $\boldsymbol{X}^{(k)} \cap \boldsymbol{N}^{(k)}$ is empty, then no root exists in $\boldsymbol{X}^{(k)}$, and also suggests the iteration scheme $\boldsymbol{X}^{(k+1)}=\boldsymbol{X}^{(k)} \cap \boldsymbol{N}^{(k)}$. In addition, if $\boldsymbol{N}^{(k)} \subset$ $\boldsymbol{X}^{(k)}$, it can been shown (e.g., Kearfoot, 1996) that there is a unique root contained in $\boldsymbol{X}^{(k)}$ and thus in $\boldsymbol{N}^{(k)}$. Thus, after computation of $\boldsymbol{N}^{(k)}$, there are three possibilities: $1 . \boldsymbol{X}^{(k)} \cap \boldsymbol{N}^{(k)}=\varnothing$, meaning 
there is no root in the current interval $\boldsymbol{X}^{(k)}$ and it can be discarded; 2. $\boldsymbol{N}^{(k)} \subset \boldsymbol{X}^{(k)}$, meaning that there is exactly one root in the current interval $\boldsymbol{X}^{(k)}$. 3. Neither of the above, meaning that no conclusion can be drawn. In the last case, if $\boldsymbol{X}^{(k)} \cap \boldsymbol{N}^{(k)}$ is sufficiently smaller than $\boldsymbol{X}^{(k)}$, then the interval-Newton test can be reapplied to the resulting intersection. Otherwise, the intersection is bisected, and the resulting two subintervals are added to the sequence of subintervals to be tested. If an interval containing a unique root has been identified, then this root can be tightly enclosed by continuing the interval-Newton iteration, which will converge quadratically to a desired tolerance (on the enclosure diameter). Alternatively, a point approximation of the root can be found by using a routine point-Newton method, starting from any point in the interval containing the unique root. This approach is referred to as an interval-Newton/generalizedbisection (IN/GB) method. At termination, when the subintervals in the sequence have all been tested, either all the real roots of $\boldsymbol{f}(\boldsymbol{x})=\mathbf{0}$ have been tightly enclosed or it is determined rigorously that no roots exist. Additional details of the IN/GB algorithm used are summarized by Schnepper and Stadtherr (1996).

An important feature of this approach is that, unlike standard methods for nonlinear equation solving that require a point initialization, the IN/GB methodology requires only an initial interval, and this interval can be sufficiently large to enclose all feasible results. In recent years, the IN/GB technique has been successfully applied to a variety of problems in chemical engineering, including phase equilibrium (Hua et al., 1998; Maier et al., 1998; Stradi et al., 2001; Xu et al., 2002), parameter estimation (Gau and Stadtherr, 2000, 2002a,b; Gau et al., 2000) and density functional theory (Maier and Stadtherr, 2001).

\section{RESULTS AND DISCUSSION}

In this section, we use the three tritrophic food chain models introduced in section 2.1 as test problems to explore the use of the IN/GB methodology for computing equilibrium states and bifurcations of equilibria. It should be noted that, since these are relatively simple models, it is possible to perform some of these computations analytically. However, since this may not be possible for more complex models, all the results presented below were computed numerically using the IN/GB technique, without any analytical short cuts. 


\subsection{Rosenzweig-MacArthur Model}

The Rosenzweig-MacArthur model used here is tritrophic, featuring a logistic prey species and hyperbolic (Holling Type II) predator and superpredator, and defined by Eqs. (7-9). Much of the literature work on the Rosenzweig-MacArthur model has focused on the enrichment paradox and chaos associated with alterations of the food chain carrying capacity (e.g., Gragnani et al., 1998). To conform to these studies and to thus provide a body of work with which to compare our results, the growth rate constant $r$ and carrying capacity $K$ were chosen as the initial set of adjustable parameters to study. Following Gragnani et al. (1998), the remaining parameters values were fixed at $a_{2}=5 / 3, b_{2}=1 / 3, e_{2}=1, d_{2}=0.4, a_{3}=0.05, b_{3}=0.5, e_{3}=1$, and $d_{3}=0.01$. Except as noted otherwise, these parameter values were used for all of the computations done here with the Rosenzweig-MacArthur model, as well as with the other two tritrophic models used.

\subsubsection{Equilibrium States}

As an initial test of the IN/GB methodology, we used it to compute equilibrium states for several sets of $K$ and $r$ values. For example consider the case of $K=1.0$ and $r=1.0$. With these values of $K$ and $r$, together with the other parameter values given above, the IN/GB method was used to solve Eq. (17) for all equilibrium states. The initial interval used for each variable was $[0,5000]$; here the upper bound is simply an arbitrarily large number. The results are shown in Table 1, along with results of stability analysis for each point. Four feasible steady states were found, all of which are unstable. Note that the values of $\boldsymbol{x}$ reported in Table 1 (as well as in Table 2 below) are rounded point representations of the interval enclosures determined by the IN/GB algorithm. For instance, the actual results computed for the first equilibrium state are the enclosures $x_{1} \in[0.819245918,0.819246099], x_{2} \in[0.124999908,0.125000008]$ and $x_{3} \in$ [9.808198838, 9.808199175]. Tighter enclosures can be determined if desired by setting a smaller tolerance for the enclosure diameter.

As another example, consider the case of $K=0.5$ and $r=1.0$. Results for this case are listed in Table 2. Again there are four feasible equilibrium states, with one stable state in this case. Both cases considered feature a steady-state with all species coexisting, along with a state including the prey and predator only, a state including the prey only, and a state for which all populations are zero. A zero population solution (solution 4) is always unstable. Physically, this 
means that the populations in any closed system whose initial populations are zero will remain zero. However, if the system is open and there is a small perturbation (increase) in the prey population, then the prey population will grow according to the logistic growth rate term until a new steady state is reached (solution 3). Such perturbations are common in aquatic environments due to flow and drift.

For a given set of $K$ and $r$ values, the computation of the equilibrium states using IN/GB is quite fast. Computation times are on the order of $0.05 \mathrm{sec}$. All computation times given here and below are for a Dell workstation running a $1.7 \mathrm{GHz}$ Intel Xeon processor and using the Intel Fortran Compiler 7.1 for Linux.

\subsubsection{Solution Branch Diagrams}

By comparing the two cases considered above, it can be seen that an effect of increasing $K$ from 0.5 to 1.0 is that the coexistence equilibrium state goes from stable to unstable. To see the changes in the equilibrium states as one parameter is varied, solution branch diagrams can be used. These are plots of both the stable and unstable steady states versus one of the parameters. Figure 1 shows the solution branch diagrams for the Rosenzweig-MacArthur model as $K$ is varied with $r=1.0$. These diagrams were generated by using the IN/GB method to repeatedly solve Eq. (17) for slightly different values of $K$, going from $K=0$ to $K=2$ in steps of $\Delta K=$ 0.001, then analyzing the stability of each solution and plotting the results (thick lines represent stable equilibria, while thin lines represent unstable equilibria). One should note that such diagrams do not give the user any information on the transient behavior of the system beyond knowledge of the stability of the equilibrium states.

Examination of Figure 1 shows that as $K$ increases there are three values of $K$ at which macroscopic changes in the system behavior (bifurcations) occur. The first of these is at $K \approx$ 0.105 . Here a new steady state appears (this is evident in the $x_{1}$ and $x_{2}$ diagrams only, as the new steady state has $x_{3}=0$ ). This is a transcritical bifurcation. In general, at a transcritical bifurcation there will be two equilibrium states that collide. However, in this case, one of the colliding states is infeasible and so does not appear on the solution branch diagram. There is another similar transcritical bifurcation at $K \approx 0.201$. Finally at $K \approx 0.768$, one of the equilibrium states (the one with all species coexisting) changes from stable to unstable. This is a Hopf bifurcation. 
Figure 1 also illustrates the paradox of enrichment as discussed by Gragnani et al. (1998), among others. This paradox states that enriching the bottom level of a food chain in order to increase the population of the top level species may, in fact, result in the decimation of the species that are wanted in greater abundance. In this case, enrichment of the food chain is modeled by increasing the prey carrying capacity $K$. The plot of superpredator population $x_{3}$ in Figure 1 illustrates that enriching the food chain results an increase in superpredator population, but this is stable only to a point. By increasing the carrying capacity beyond this critical point, the stable steady-state becomes unstable and enrichment may become counterproductive. Solution branch diagrams such as Figure 1 can be very easily and automatically generated using the IN/GB methodology, with certainty that all equilibrium states (solution branches) have been found. Two other solution branch diagrams were computed. Figure 2 shows the case for $r=0.5$, and Figure 3 for $r=0.4$.

Figure 2 illustrates some bifurcation behavior similar to Figure 1, but with distinct differences. Here as $K$ is increased, the system undergoes a transcritical bifurcation in which a previously infeasible equilibrium state becomes feasible, colliding with another equilibrium state and exchanging stabilities. This is followed by a Hopf bifurcation, which occurs for an equilibrium state with a positive prey and predator population, but with a zero superpredator population. Therefore, this is a planar Hopf bifurcation, as the bifurcation is occurring in a subset of the state space of the model. At $K \approx 0.872$, a fold bifurcation occurs and two new steady states appear (this is evident only in the plots of $x_{1}$ and $x_{3}$, as the new steady states have the same value of $x_{2}$ ). Finally there are two Hopf bifurcations. One can observe that there is no continuity between the equilibrium that undergoes the first (planar) Hopf bifurcation and the equilibrium that undergoes the second two (non-planar) Hopf bifurcations. From the plot of $x_{3}$, it is evident that the region of stable coexistence of all three populations at equilibrium is in the narrow interval of $K$ values between the second two Hopf bifurcation points. However, in this region, the trend of the enrichment paradox is apparent.

Looking at Figure 3, one can see that the slight change made in the prey growth rate constant $r$ leads to a significant change in system behavior. The narrow band of stability in Figure 2 that allows all three species to coexist no longer exists in Figure 3 . Thus, at a sufficiently low prey growth rate, no superpredators can thrive in a stable population. It is also very interesting to note that in Figure 3 the fold bifurcation results in two equilibrium states 
(solution branches) that do not intersect other branches (this is true even for larger values of $K$ than shown on the plot). Such isolated solution branches (isola) can be very difficult to find using continuation methods, especially for more complex models in which their existence may not be suspected. However, using the IN/GB approach, isolated branches are easily found. In fact, there is a mathematical and computational guarantee that they will be found.

\subsubsection{K vs. $r$ Bifurcation Diagram}

For fixed $r$, the values of $K$ and $\boldsymbol{x}$ at which the bifurcations of equilibria observed above occur can be computed directly by solving the appropriate augmented systems, namely Eqs. (2021) for fold and transcritical bifurcations and Eqs. (22-23) for Hopf bifurcations. In a $K$ vs. $r$ bifurcation diagram the values of $K$ at which the bifurcations occur are plotted as a function of $r$. Such a diagram was generated here by using the IN/GB method to repeatedly solve the augmented systems for $K$ and $\boldsymbol{x}$ for slightly different values of $r$, going from $r=0$ to $r=2$ in steps of $\Delta r=0.005$. There may be some values of $r$ for which one of the augmented systems has an infinite number of solutions for $K$ (i.e., a vertical line on the $K$ vs. $r$ diagram). This case cannot be handled directly by the IN/GB technique, or could be missed entirely by the stepping in $r$. Thus, to ensure that all of the bifurcations are found, it is necessary to also scan in the $K$ direction. That is, the IN/GB method was also used to repeatedly solve the augmented systems for $r$ and $\boldsymbol{x}$ for slightly different values of $K$, in this case going from $K=0$ to $K=2$ in steps of $\Delta K$ $=0.005$. To locate codimension-two bifurcations (double-fold and fold-Hopf), the IN/GB method was used to solve the doubly augmented system given by Eqs. (25-27) for $K, r$ and $\boldsymbol{x}$. The initial intervals used for the components of $\boldsymbol{x}$ were again [0,5000] and for the parameters $K$ and $r$ were [0,2]. The average CPU time for each solution of Eqs. (20-21) for fold and transcritical bifurcations was about 0.6 seconds, and for each solution of Eqs. (22-23) for Hopf bifurcations was about 1.4 seconds. Solving Eqs. (25-27) for codimension-two bifurcations required about 39 seconds.

Figure 4 shows the $K$ vs. $r$ bifurcation diagram generated for the Rosenzweig-MacArthur tritrophic food chain model using the IN/GB method. Fold and transcritical of equilibria curves were both found, and are labeled FE and TE respectively. Hopf bifurcation curves were also found, and are labeled $\mathrm{H}$ or $\mathrm{H}_{\mathrm{p}}$ (for planar Hopf). A single fold-Hopf bifurcation was located; this point is represented as an open diamond and labeled FH (no double-fold bifurcations were 
found). This bifurcation diagram corresponds exactly with the known $K$ vs. $r$ bifurcation diagram for this model, as reported by Gragnani et al. (1998). This confirms the utility and accuracy of the IN/GB algorithm for computing bifurcation of equilibria diagrams. Bifurcation diagrams such as Figure 4 can be very easily and automatically generated using the IN/GB methodology, with complete certainty that all bifurcation curves have been found. Two other bifurcation diagrams were computed, $d_{2}$ vs. $K$ and $r$ vs. $d_{2}$.

\subsection{4 $d_{2}$ vs. $K$ Bifurcation Diagram}

Using the same procedure as described above, a $d_{2}$ vs. $K$ bifurcation diagram for the Rosenzweig-MacArthur model was generated. The predator death rate constant $d_{2}$ is now a free parameter, and $r$ is now a fixed parameter set at $r=1$. The average CPU time for each solution of Eqs. (20-21) for fold and transcritical bifurcations was about 0.8 seconds, and for each solution of Eqs. (22-23) for Hopf bifurcations was about 2.1 seconds. Solving Eqs. (25-27) for codimension-two bifurcations required about 31 seconds. The resulting bifurcation diagram is shown in Figure 5. This diagram illustrates that at a constant prey carrying capacity and growth rate constant $(r=1)$, increasing or decreasing the predator death rate will cause macroscopic changes in system behavior. For relatively small values of $K$, there are two transcritical bifurcations that occur as $d_{2}$ is changed, and for larger values of $K$ there are also two Hopf bifurcations. No double-fold or fold-Hopf codimension-two bifurcations were found. In order to more closely observe these changes in behavior, solution branch diagrams were generated using IN/GB for the case of $K=1$. Figure 6 gives the solution branch diagrams for $\boldsymbol{x}$ as $d_{2}$ is varied from 0 to 2 .

Based on the bifurcation diagram at $K=1$, we would expect that as $d_{2}$ is increased from 0 to 2, there should be observed first a Hopf bifurcation (the planar Hopf is not observed in this case, due to the sign of the third eigenvalue) and then two transcritical bifurcations. This is what is in fact seen in Figure 6. These diagrams illustrate that there is a minimum predator death rate constant $d_{2}$ that results in stable system behavior. At low predator death rates, the system is unstable and likely exhibits cycles of population booms and busts. As the predator death rate increases, enough predators are dying off at any given time to prevent the cycles from occurring, and the cycles collapse to a stable steady-state in a Hopf bifurcation.

These results also give a sense of the effects of releasing a toxin that specifically targets the predator trophic level, and increases the predator death rate constant. Prior to examining 
these diagrams, one would expect that such a release would have an impact on both the predator and the superpredator populations. The plot of $x_{3}$ in Figure 6 shows that increasing the predator death rate constant causes a linear decrease in the stable superpredator biomass. However, according to the plot of $x_{2}$ in Figure 6, the stable predator population is not affected until the superpredator population reaches zero. Though these results may seem somewhat counterintuitive, they are indicative of the complex interactions that may occur in food chains. An ecotoxin released at a very low concentration could affect organisms at different trophic levels to varying degrees. For the case considered here, one might observe an impact on the superpredator population and thus assume that the effect of the ecotoxin was at that level, even though the actual impact is on the predator level. Using models such as this one can obtain insights into the impacts of an ecotoxin that might not otherwise be apparent.

\subsection{5 $r$ vs. $d_{2}$ Bifurcation Diagram}

Again using the IN/GB methodology, an $r$ vs. $d_{2}$ bifurcation diagram for the RosenzweigMacArthur model was generated, with $K$ fixed at $K=1$. This set of free parameters is of interest since both could be affected by an ecotoxin. Since the prey growth rate constant represents the net growth (accounting for both birth and natural death), an ecotoxin affecting the prey trophic level could decrease the prey growth rate. For this problem, the average CPU time for each solution of Eqs. (20-21) for fold and transcritical bifurcations was about 2.4 seconds, and for each solution of Eqs. (22-23) for Hopf bifurcations was about 2.2 seconds. Solving Eqs. (25-27) for codimension-two bifurcations required about 220 seconds. The resulting bifurcation diagram is shown in Figure 7.

Figure 7 displays a wide variety of bifurcation behavior, including a codimension-two fold-Hopf bifurcation. This diagram illustrates that changing either the prey growth rate constant or the predator death rate constant can cause macroscopic changes in system behavior. Two solution branch diagrams were generated in IN/GB to more closely examine the changes in species biomass as the parameter variables are changed. Figure 8 is the solution branch diagram as $d_{2}$ is changed at a constant $r=0.5$, and Figure 9 is the solution branch diagram as $r$ is changed at a constant $d_{2}=0.4$.

The solution branch diagrams in Figure $8(r=0.5 ; K=1.0)$ illustrate behavior somewhat similar to the solution branch diagrams illustrated in Figure $6(r=1.0 ; K=1.0)$, with important differences. First, in Figure 8 a third transcritical bifurcation is observed, at a value of $d_{2}$ very 
close to the Hopf bifurcation. Also, compared to Figure 6, the Hopf bifurcation now occurs at a lower value of $d_{2}$, as does the point where the system can no longer support superpredators and they become extinct. However, the point at which the predator population becomes extinct does not change, nor does the rate of superpredator decline. Therefore, with a decrease in the prey growth rate constant from $r=1.0$ to $r=0.5$, the system actually has a wider range of $d_{2}$ that results in a stable system, and a wider range of $d_{2}$ in which all three species can coexist.

Figure 9 illustrates the effect of increasing the prey growth rate constant on a system with constant carrying capacity $K=1.0$ and constant predator death rate constant $d_{2}=0.4$. These solution branch diagrams tie together Figure 4 ( $K$ vs. $r$ at constant $\left.d_{2}=0.4\right)$ and Figure 7 ( $r$ vs. $d_{2}$ at constant $K=1.0)$ in that they are evaluated at a parameter set $\left(K=1.0 ; d_{2}=0.4\right)$ common to both diagrams. As $r$ increases, the solution branch diagrams illustrated in Figure 9 exhibit a fold bifurcation, then a Hopf bifurcation, followed very closely by a transcritical bifurcation, and finally another Hopf bifurcation. The location of these bifurcations can be verified by both Figure 4 (following the line $K=1$ upwards) and Figure 7 (following the line $d_{2}=0.4$ upwards). This example and those above are useful in confirming that the IN/GB methodology is indeed successfully computing all equilibrium states and bifurcations of equilibria for this model. The solution branch diagrams of Figure 9 show a single region of stability for the model, and in this region all three species coexist. In this region, increasing the prey growth rate constant causes an increase in prey and superpredator population, but this occurs only to a point. This type of phenomenon is similar to the paradox of enrichment. As the prey species replaces its population more quickly, more organisms are able to thrive within the food chain, but eventually if the prey population grows too quickly, the system becomes unstable.

\subsection{Tritrophic Model with Sigmoidal Predator and Superpredator Responses}

In view of the success in applying the IN/GB methodology to generate bifurcation diagrams and solution branch diagrams for the Rosenzweig-MacArthur model, the methodology was tested on two other food chain models. The first of these is the tritrophic model with sigmoidal (Holling Type III) predator and superpredator functional responses, as given by Eqs (10-12). 


\subsubsection{Bifurcation Diagram}

The dynamics of this system have received only limited study (Turchin, 2003; Yodzis, 1989), and there are apparently no published bifurcation diagrams for it. Following the procedures outlined above, the IN/GB methodology was applied to compute the bifurcation diagram for the case of $r$ and $K$ as free parameters. The average CPU time for each solution of Eqs. (20-21) for fold and transcritical bifurcations was about 3.6 seconds, and for each solution of Eqs. (22-23) for Hopf bifurcations was about 7.9 seconds. Solving Eqs. (25-27) for codimension-two bifurcations (double-fold or fold-Hold) required about 71 seconds. The resulting bifurcation diagram is shown in Figure 10.

At least for the range of parameters studied, no Hopf bifurcations were found, and no double-fold or fold-Hopf codimension-two bifurcations were found. Note that the range of prey carrying capacity values studied was increased to $[0,4]$ in order to more closely examine the pair of fold of equilibria curves discovered. These curves are isolated from the transcritical of equilibria bifurcation curves in the parameter-state space in which Eqs. (20-21) are solved, and so could be difficult to detect using continuation methods. The intersection of the two fold of equilibria bifurcation curves without the occurrence of a double-fold bifurcation suggests that this point is a cusp bifurcation. Note that this type of codimension-two bifurcation cannot be found by solving Eqs. (25-27). In order to investigate the behavior of the system near the cusp, solution branch diagrams were generated for the case $r=0.7$ using the IN/GB methodology.

\subsubsection{Solution Branch Diagrams}

A set of solution branch diagrams was generated for this model that examines the effect of increasing the prey carrying capacity $K$ on the biomasses of the three trophic levels while holding $r$ constant at a value of 0.7 . This value was chosen to intersect with the fold bifurcation curves close to the cusp. Figure 11 gives the solution branch diagrams. These illustrate the crossing of two transcritical bifurcations followed by two fold bifurcations. One equilibrium created by the first fold bifurcation collides with the equilibrium that appears in the second transcritical bifurcation and the two mutually annihilate in the second fold bifurcation, forming an $\mathrm{S}$ shaped curve typical of behavior near a cusp bifurcation. Note that there is a region where two stable steady-states exist in which all three species can coexist; this region also contains an unstable-steady state (this is not seen in the plot for $x_{2}$ since all three of these solutions have the 
same $x_{2}$ value). It also appears, according to this model, that the enrichment paradox does not hold for systems of generalist predators, as increasing $K$ does not ultimately result in an unstable system. However, it should be noted that in this system the two fold bifurcations are catastrophic because they result in an abrupt change in system behavior. For instance, if the system is at the stable high-population (prey and superpredator) equilibrium state, and the prey carrying capacity $K$ is decreasing, then, at the leftmost fold bifurcation, this state suddenly disappears and is replaced by a low-population equilibrium state. The transient behavior by which the new lowpopulation state is approached is not investigated here.

\subsection{Tritrophic Model with Hyperbolic Predator and Sigmoidal Superpredator Responses}

The last of the food chain models used as a test problem here is the tritrophic model with a hyperbolic (Holling Type II) predator response and a sigmoidal (Holling Type III) superpredator response, as given by Eqs (13-15).

\subsubsection{Bifurcation Diagram}

This model has apparently received little, if any, previous study. Using a hyperbolic (specialist) predator and a sigmoidal (generalist) superpredator is justifiable in that organisms that are higher up on a food chain tend to have more diversity in the types of organisms that compose their diets. Again the IN/GB methodology was applied to compute the bifurcation diagram for the case of $r$ and $K$ as free parameters. The average CPU time for each solution of Eqs. (20-21) for fold and transcritical bifurcations was about 2.1 seconds, and for each solution of Eqs. (22-23) for Hopf bifurcations was about 3.8 seconds. Solving Eqs. (25-27) for codimension-two bifurcations (double-fold or fold-Hold) required about 62 seconds. The resulting bifurcation diagram is shown in Figure 12.

The bifurcation diagram illustrates a range of features, including fold and transcritical of equilibria bifurcations, Hopf bifurcations, and a codimension-two bifurcation point classified as a fold-Hopf bifurcation. The fold and transcritical bifurcation curves appear to be quite similar to those seen in the Rosenzweig-MacArthur model, however the Hopf bifurcation behavior is quite different in that the Hopf curve that originates at the fold-Hopf bifurcation point does not double back in the diagram for this model. Also the fold-Hopf point occurs at a significantly larger value of $r$. 


\subsubsection{Solution Branch Diagrams}

Using the IN/GB methodology, solution branch diagrams were generated for this model that examine the effect of increasing the prey carrying capacity $K$ on the biomasses in the three trophic levels, while holding $r$ constant at a value of 1.0. These diagrams are shown in Figure 13. The solution branch diagrams illustrate a transcritical bifurcation followed by a Hopf bifurcation and then a fold bifurcation. An interesting feature to note is that the Hopf bifurcation that causes a change in system stability is, in fact, a planar Hopf bifurcation. A second Hopf bifurcation is encountered with no change in system stability. This Hopf bifurcation is nonplanar, but a change in stability does not occur as the sign of the third eigenvalue is already positive. As one equilibrium created in the fold bifurcation approaches $K=2.0$, it grows close to a transcritical bifurcation. This model displays a region of instability between the Hopf and fold bifurcations. However, the model does not exhibit behavior in accordance with the enrichment paradox. While increasing the prey carrying capacity does take the system through a region of instability, the presence of a generalist superpredator causes the system to be stable for larger values of $K$, at least for the parameter values at which this diagram was generated.

\subsection{Computational Performance}

Average computation times are given above for single solutions of the appropriate nonlinear equation systems for determination of equilibrium states, codimension-one bifurcations and codimension-two bifurcations. To generate an equilibrium solution branch diagram or a bifurcation diagram requires that these equation systems be solved multiple times. For instance, a solution branch diagram generated over a parameter range $[0,2]$ with a step size of 0.001 would require 2000 solutions of Eq. (17) for the equilibrium states. With a solution time on the order of 0.05 seconds for an individual system, this means that the entire solution branch diagram requires roughly 100 seconds of computation time. Bifurcation diagrams are more costly since both Eqs. (20-21) and Eqs (22-23) must be solved repeatedly, and Eqs. (25-27) once. For example, the $K$ vs. $r$ bifurcation diagram for the Rosenzwieg-MacArthur model requires about 1640 seconds of computation time. We do not consider computational effort on this order to be unreasonable, especially since the methodology used provides a guarantee of reliability. Furthermore, since the diagrams can be generated automatically, without user intervention to 
deal with initialization issues, the actual elapsed time to generate a bifurcation diagram for a new model may actually be significantly less than when initialization-dependent methods are used.

Since all of the nonlinear equation systems that must be solved to generate a diagram are independent of each other, one obvious way to improve computational performance is to use parallel computing. Distribution of the independent equation systems across multiple processors will result in essentially linear speedup. Furthermore, the IN/GB methodology itself can be readily parallelized; for example, Gau and Stadtherr (2002c) have described an MPI-based implementation of IN/GB that provides very efficient processor utilization. The serial performance of the methodology can also be easily improved by using additional tools from interval analysis, including constraint propagation and the exploitation of function properties (e.g., monotonicity) in evaluating interval extensions. The work of Maier and Stadtherr (2001) on an application arising in the modeling of phase transitions in nanopores demonstrates the use of these types of techniques.

\section{CONCLUDING REMARKS}

Using several examples drawn from three different tritrophic food chain models, we have demonstrated a new methodology for computing all equilibrium states and bifurcations of equilibria (fold, transcritical, Hopf, double-fold and fold-Hopf). This technique is based on interval analysis, in particular an interval-Newton/generalized bisection (IN/GB) approach. Using this methodology it was possible to easily and automatically, without any need for initialization or a priori insight into expected system behavior, generate complete solution branch diagrams and bifurcation diagrams. Furthermore, this could be done with certainty, since the technique provides a mathematical and computational guarantee that all solutions to a system of nonlinear equations are enclosed. Since this technique is essentially initialization independent, it can provide a powerful alternative to traditional continuation methods, which in general are initialization dependant and thus may not be completely reliable. Although the systems studied here were relatively simple, we anticipate that the methodology used can be applied to larger and more complex problems, as well as in the analysis of other dynamical systems of interest in chemical engineering. 


\section{Acknowledgements}

This work was supported in part by the Department of Education Graduate Assistance in Areas of National Needs (GAANN) Program under Grant \#P200A010448, and by the State of Indiana $21^{\text {st }}$ Century Research and Technology Fund under Grant \#909010455. Additional support has been provided by a University of Notre Dame Schmitt Fellowship (CRG) and by a University of Notre Dame Center for Applied Mathematics Undergraduate Summer Fellowship (MPS).

\section{References}

Abrams, P. A., \& Roth, J. D. (1994). The effects of enrichment of three-species food chains with non-linear functional responses. Ecology 75, 1118-1130.

Brennecke, J. F., \& Maginn, E. J. (2001). Ionic liquids: Innovative fluids for chemical processing. AIChE Journal 47, 2384-2389.

De Feo, O., \& Rinaldi, S. (1997). Yield and dynamics of tritrophic food chains. American Naturalist 150, 328-345.

Doedel, E. J., Champneys, A. R., Fairgrieve, T. F., Kuznetsov, Y. A., Sandstede, B. and X.J Wang (1997). AUTO97: Continuation and bifurcation software for ordinary differential equations. Department of Computer Science, Concordia University, Montreal, Canada (see also http://indy.cs.concordia.ca/auto/bib)

Freemantle, M. (2002). Meeting briefs: Ionic liquids separated from mixtures by $\mathrm{CO}_{2}$. Chemical \& Engineering News 80 (36), 44-45.

Gau, C.-Y., \& Stadtherr, M. A. (2000). Reliable nonlinear parameter estimation using interval analysis: Error-in-variables approach. Computers \& Chemical Engineering 24, 631-638.

Gau, C.-Y., \& Stadtherr, M. A. (2002a). Deterministic global optimization for error-in-variables parameter estimation. AIChE Journal 48, 1192-1197.

Gau, C.-Y., \& Stadtherr, M. A. (2002b). Deterministic global optimization for data reconciliation and parameter estimation using error-in-variables approach. In Recent Developments in Optimization and Optimal Control in Chemical Engineering (ed. R. Luus), Transworld Research, pp. 1-17. 
Gau, C.-Y., \& Stadtherr, M. A. (2002c). Dynamic load balancing for parallel interval-Newton using message passing. Computers \& Chemical Engineering 26, 811-825.

Gau, C.-Y., Brennecke, J. F., \& Stadtherr, M. A. (2000). Reliable nonlinear parameter estimation in VLE modeling. Fluid Phase Equilibr., 168 (1): 1-18.

Gragnani, A., De Feo, O., \& Rinaldi, S., (1998). Food chains in the chemostat: Relationships between mean yield and complex dynamics. Bulletin of Mathematical Biology 60, 703719.

Hansen, E. R. (1992). Global Optimization Using Interval Analysis, Marcel Dekker, New York. Hastings, A., \& Powell, T. (1991). Chaos in a three-species food chain. Ecology 72, 896-903.

Hua, J. Z., Brennecke, J. F., \& Stadtherr, M. A. (1998). Enhanced interval analysis for phase stability: Cubic equation of state models. Industrial \& Engineering Chemistry Research 37, 1519-1527.

Holling, C. S. (1959). Some characteristics of simple types of predation and parasitism. Canadian Entomologist 91, 385-398.

Kearfott, R. B. (1996). Rigorous Global Search: Continuous Problems, Kluwer, Dordrecht, The Netherlands.

Klebanoff, A., \& Hastings, A. (1994). Chaos in three-species food chains. Journal of Mathematical Biology 32, 427-451.

Kuznetsov, Y. A. (1998). Elements of Applied Bifurcation Theory, Springer-Verlag, New York.

Kuznetsov, Y. A., \& Rinaldi, S. (1996). Remarks on food chain dynamics. Mathematical Biosciences 134, 1-33.

Jastorff, B., Störmann, R., Ranke, J., Mölter, K., Stock, F., Oberheitmann, B., Hoffmann, W., Hoffmann, J., Nüchter, M., Ondruschka, B., \& Filser, J. (2003). How hazardous are ionic liquids? Structure-activity relationships and biological testing as important elements for sustainability evaluation. Green Chemistry 5, 136-142.

Maier, R.W., \& Stadtherr, M. A. (2001). Reliable density-functional-theory calculations of adsorption in nanoscale pores. AIChE Journal 47, 1874-1884.

Maier, R.W., Brennecke, J. F., \& Stadtherr, M. A. (1998). Reliable computation of homogeneous azeotropes. AIChE Journal 44, 1745-1755.

Moghadas, S. M., \& Gumel, A. B. (2003). Dynamical and numerical analyses of a generalized food-chain model. Applied Mathematics and Computation, 142 (1): 35-49. 
Moore, R. E. (1966). Interval Analysis, Prentice-Hall, Englewood Cliffs, NJ.

Neumaier, A. (1990). Interval Methods for Systems of Equations, Cambridge Univ. Press, Cambridge, U.K.

Stradi, B., Brennecke, J. F., Kohn, J. P., \& Stadtherr, M. A. (2001). Reliable computation of mixture critical points. AIChE Journal 47, 212-221.

Turchin, P. (2003). Complex Population Dynamics: A Theoretical/Empirical Synthesis.

Princeton Univ. Press, Princeton, NJ.

van Coller, L. (1997). Automated techniques for the qualitative analysis of ecological models: Continuous models. Conserv. Ecol. [online], 1 (1), 5. URL: http://www.consecol.org/vol1/iss1/art5.

Xu, G., Brennecke, J. F., \& Stadtherr, M. A. (2002). Reliable computation of phase stability and equilibrium from the SAFT equation of state. Industrial \& Engineering Chemistry Research 41, 938-952 (2002).

Yodzis, P. (1989). Introduction to Theoretical Ecology. Harper \& Row, New York. 
Table 1: Computed equilibrium states for the Rosenzweig-MacArthur model with $K=1.0$ and $r$ $=1.0$.

\begin{tabular}{ccccc}
\hline Solution \# & $\mathbf{x}_{\mathbf{1}}$ & $\mathbf{x}_{\mathbf{2}}$ & $\mathbf{x}_{\mathbf{3}}$ & Stability \\
1 & 0.819 & 0.125 & 9.808 & Unstable \\
2 & 0.105 & 0.235 & 0 & Unstable \\
3 & 1.000 & 0 & 0 & Unstable \\
4 & 0 & 0 & 0 & Unstable \\
\hline
\end{tabular}


Table 2: Computed equilibrium states for the Rosenzweig-MacArthur model with $K=0.5$ and $r$ $=1.0$.

\begin{tabular}{ccccc}
\hline Solution \# & $\mathbf{x}_{\mathbf{1}}$ & $\mathbf{x}_{\mathbf{2}}$ & $\mathbf{x}_{\mathbf{3}}$ & Stability \\
1 & 0.347 & 0.125 & 5.624 & Stable \\
2 & 0.105 & 0.208 & 0 & Unstable \\
3 & 0.500 & 0 & 0 & Unstable \\
4 & 0 & 0 & 0 & Unstable \\
\hline
\end{tabular}




\section{List of Figures}

Figure 1. Solution branch diagrams illustrating the change in species biomass with the change in the prey carrying capacity $K$ for the Rosenzweig-MacArthur model. From left to right: prey, predator, and superpredator biomasses. $r=1.0$ for all three plots.

Figure 2. Solution branch diagrams illustrating the change in species biomass with the change in the prey carrying capacity $K$ for the Rosenzweig-MacArthur model. From left to right: prey, predator, and superpredator biomasses. $r=0.5$ for all three plots.

Figure 3. Solution branch diagrams illustrating the change in species biomass with the change in the prey carrying capacity $K$ for the Rosenzweig-MacArthur model. From left to right: prey, predator, and superpredator biomasses. $r=0.4$ for all three plots.

Figure 4. Bifurcation diagram of $K$ (prey carrying capacity) vs. $r$ (prey growth rate constant) for the Rosenzweig-MacArthur model.

Figure 5. Bifurcation diagram of $K$ (prey carrying capacity) vs. $d_{2}$ (predator death rate constant) for the Rosenzweig-MacArthur model. $r=1.0$.

Figure 6. Solution branch diagrams illustrating the change in species biomass with the change in the predator death rate constant $d_{2}$ for the Rosenzweig-MacArthur model. From left to right: prey, predator, and superpredator biomasses. $\mathrm{K}=1.0$ and $\mathrm{r}=1.0$ for all three plots.

Figure 7. Bifurcation diagram of $d_{2}$ (predator death rate constant) vs. $r$ (prey growth rate constant) for the Rosenzweig-MacArthur model. $K=1.0$.

Figure 8. Solution branch diagrams illustrating the change in species biomass with the change in the predator death rate constant $d_{2}$ for the Rosenzweig-MacArthur model. From left to right: prey, predator, and superpredator biomasses. $K=1.0$ and $r=0.5$ for all three plots.

Figure 9. Solution branch diagrams illustrating the change in species biomass with the change in the prey growth rate constant $r$ for the Rosenzweig-MacArthur model. From left to right: prey, predator, and superpredator biomasses. $K=1.0$ and $d_{2}=0.4$ in all three plots.

Figure 10. Bifurcation diagram of $K$ (prey carrying capacity) vs. $r$ (prey growth rate constant) for the tri-trophic, sigmoidal response model.

Figure 11. Solution branch diagrams illustrating the change in species biomass with the change in the prey carrying capacity $K$ for the tri-trophic, sigmoidal response model. From left to right: prey, predator, and superpredator biomasses. $r=0.7$ for all three plots. 
Figure 12. Bifurcation diagram of $K$ (prey carrying capacity) vs. $r$ (prey growth rate constant) for the tri-trophic model with a hyperbolic predator and a sigmoidal superpredator.

Figure 13. Solution branch diagrams illustrating the change in species biomass with the change in the prey carrying capacity $K$ for the tri-trophic model with a hyperbolic predator and a sigmoidal superpredator. From left to right: prey, predator, and superpredator biomasses. $r$ $=1.0$ for all three plots. 


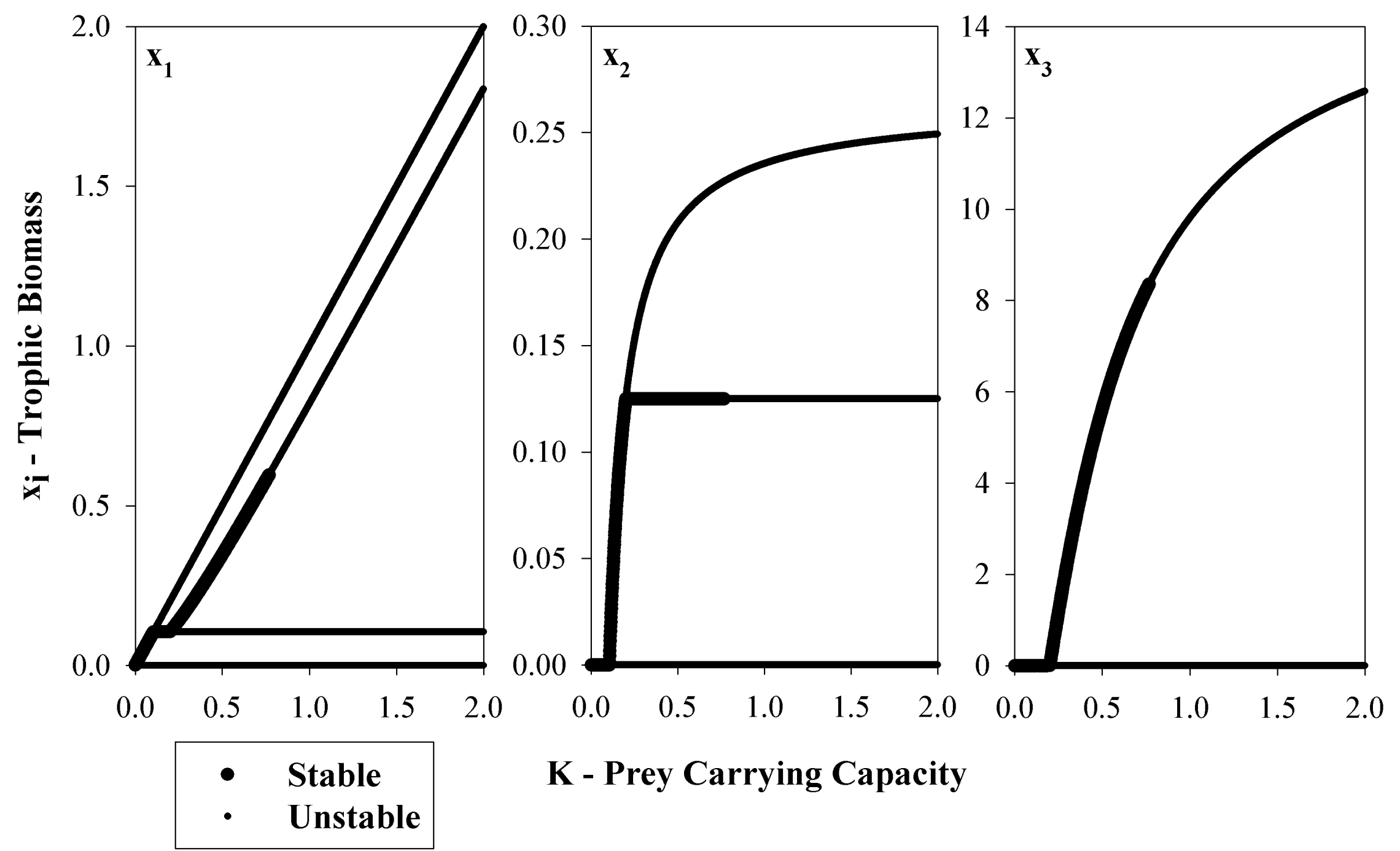

Figure 1. Solution branch diagrams illustrating the change in species biomass with the change in the prey carrying capacity $K$ for the Rosenzweig-MacArthur model. From left to right: prey, predator, and superpredator biomasses. $r=1.0$ for all three plots. 

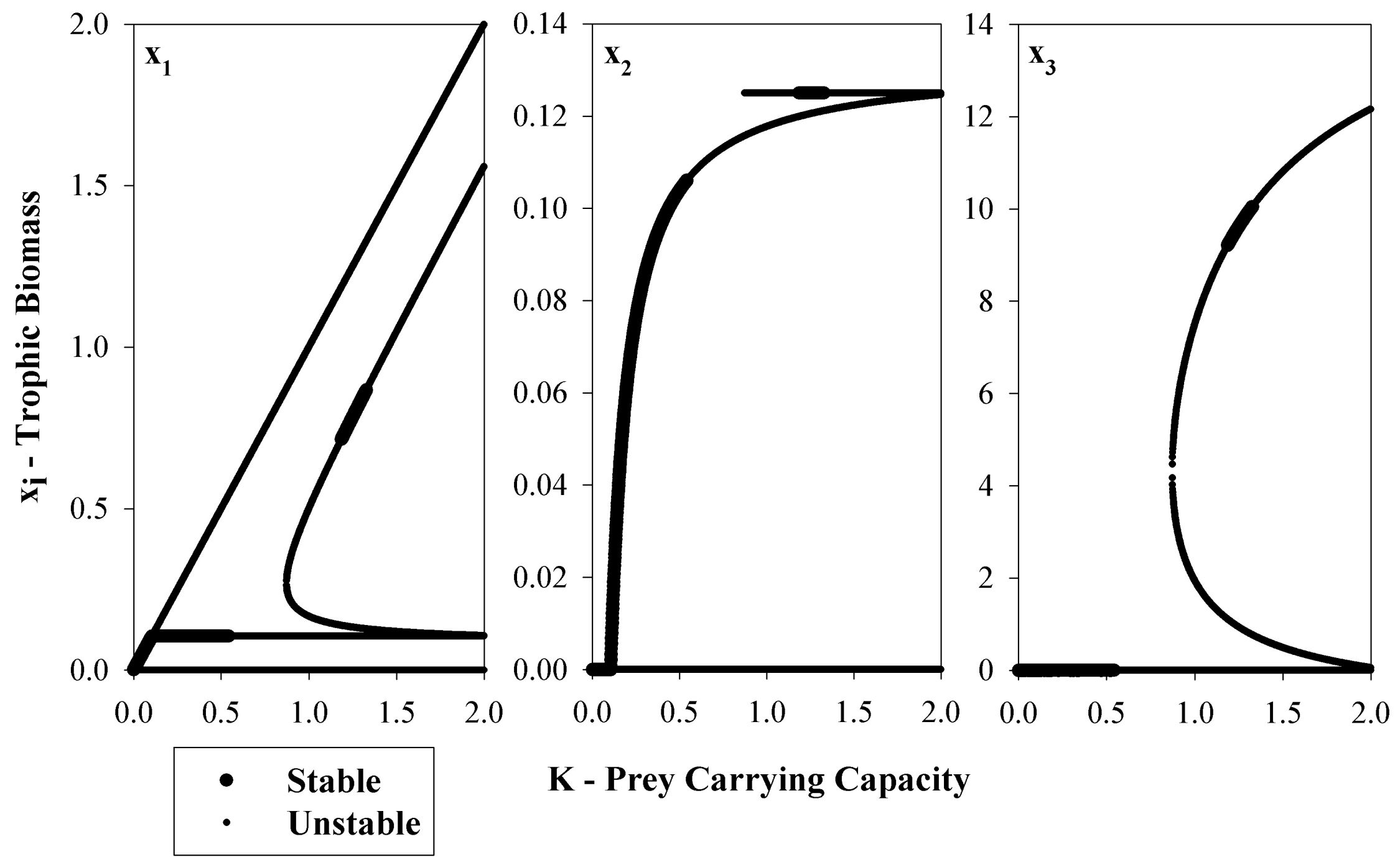

Figure 2. Solution branch diagrams illustrating the change in species biomass with the change in the prey carrying capacity $K$ for the Rosenzweig-MacArthur model. From left to right: prey, predator, and superpredator biomasses. $r=0.5$ for all three plots. 


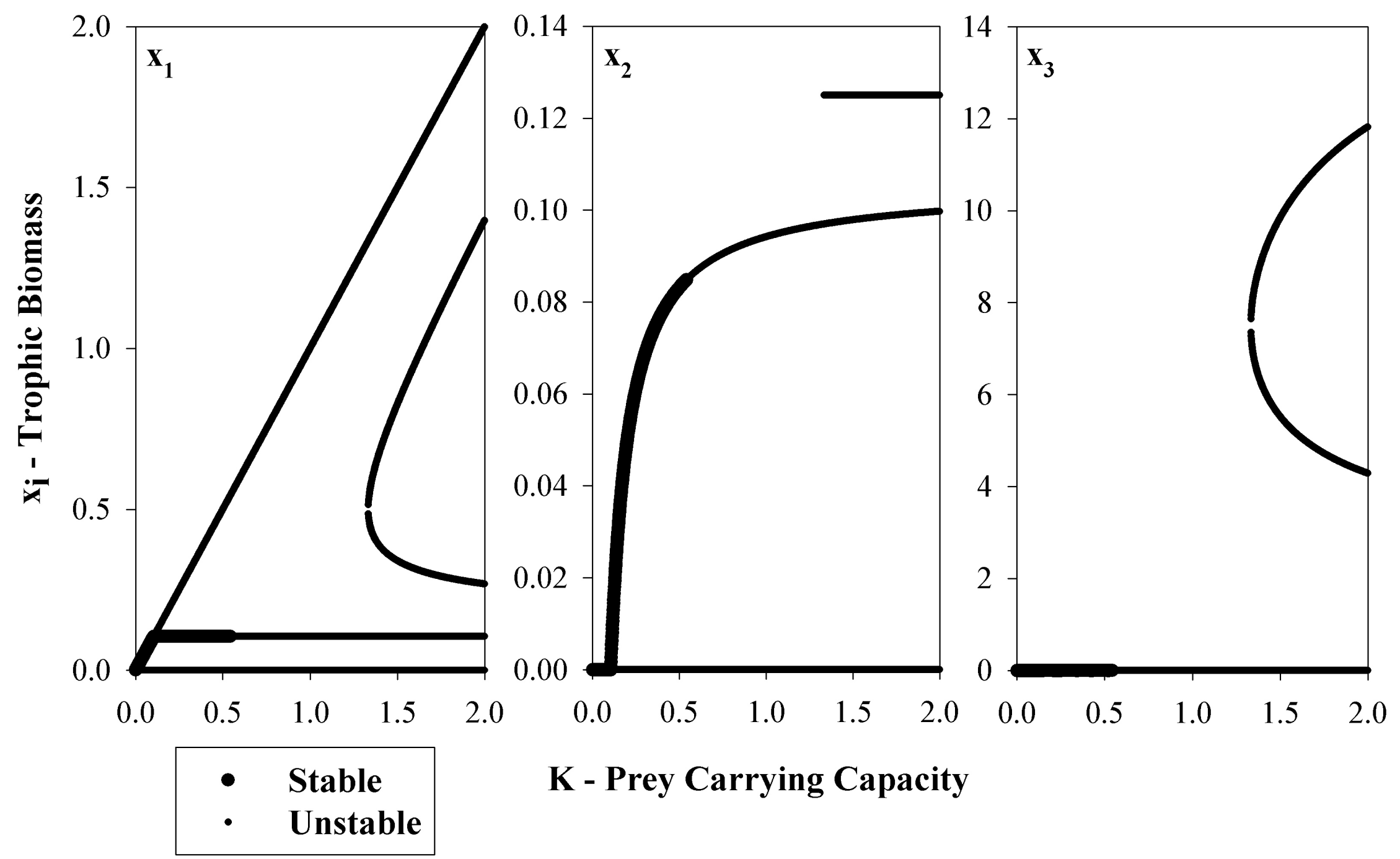

Figure 3. Solution branch diagrams illustrating the change in species biomass with the change in the prey carrying capacity $K$ for the Rosenzweig-MacArthur model. From left to right: prey, predator, and superpredator biomasses. $r=0.4$ for all three plots. 


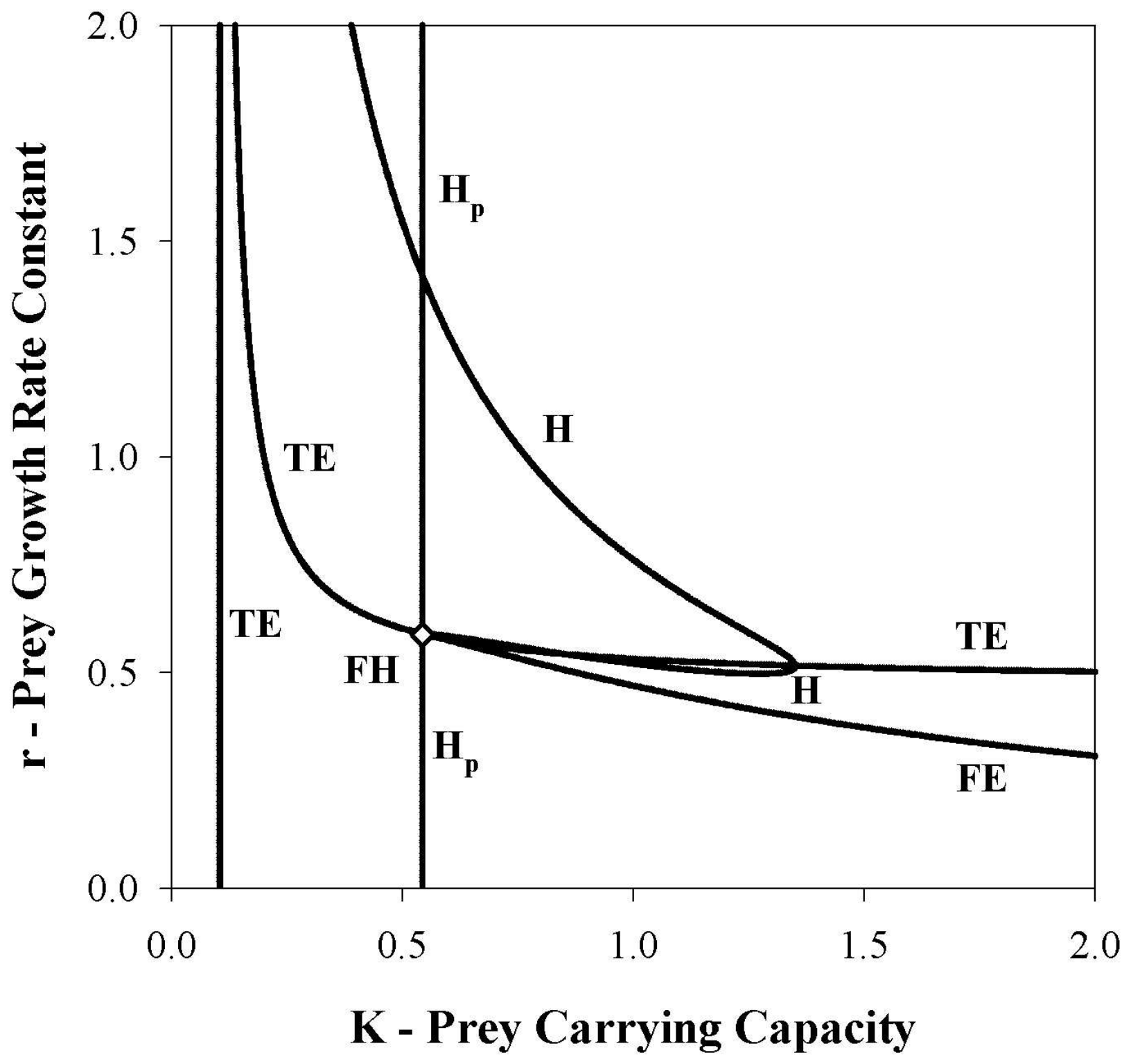

Figure 4. Bifurcation diagram of $K$ (prey carrying capacity) vs. $r$ (prey growth rate constant) for the Rosenzweig-MacArthur model. 


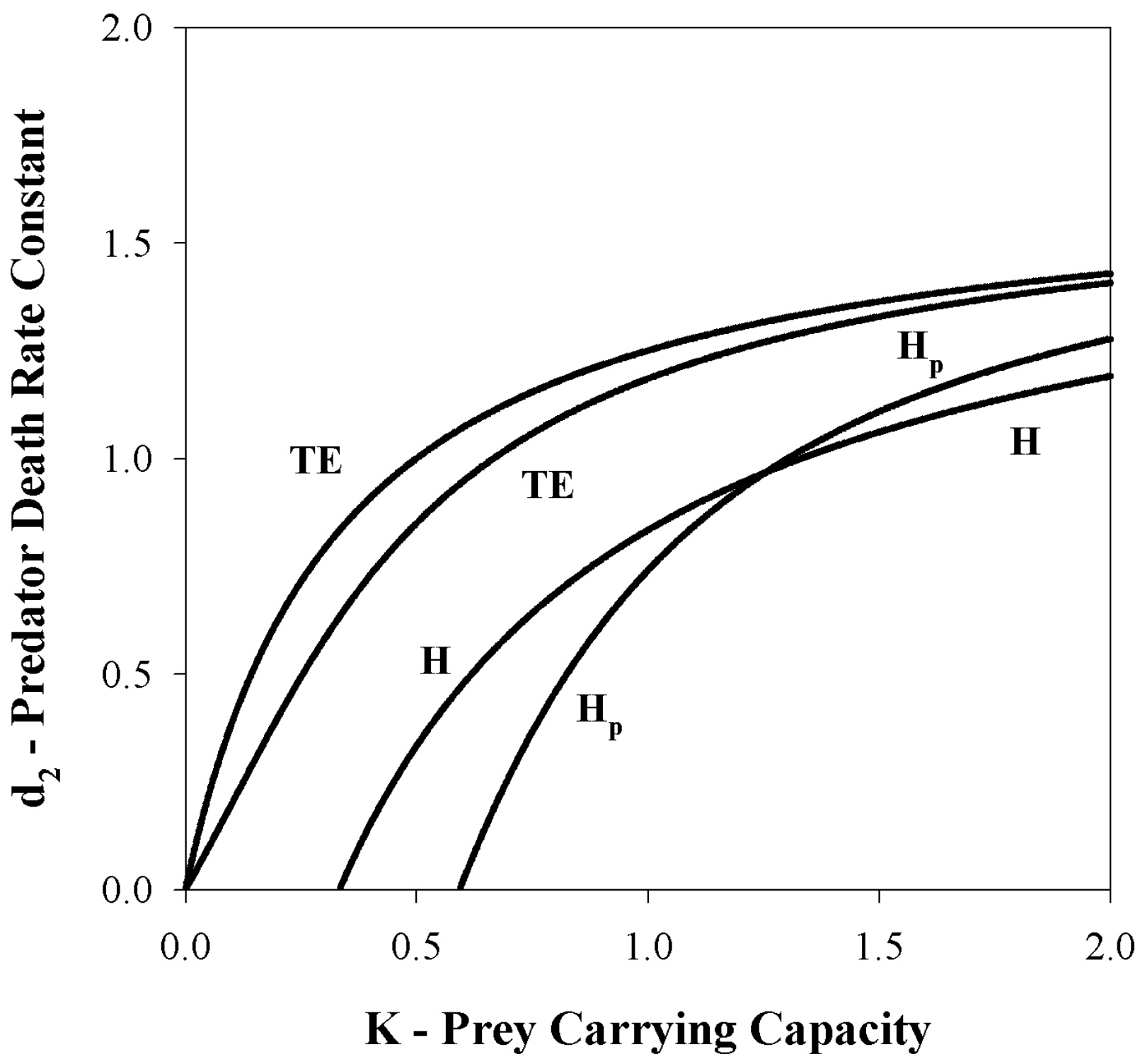

Figure 5. Bifurcation diagram of $K$ (prey carrying capacity) vs. $d_{2}$ (predator death rate constant) for the Rosenzweig-MacArthur model. $r=1.0$. 


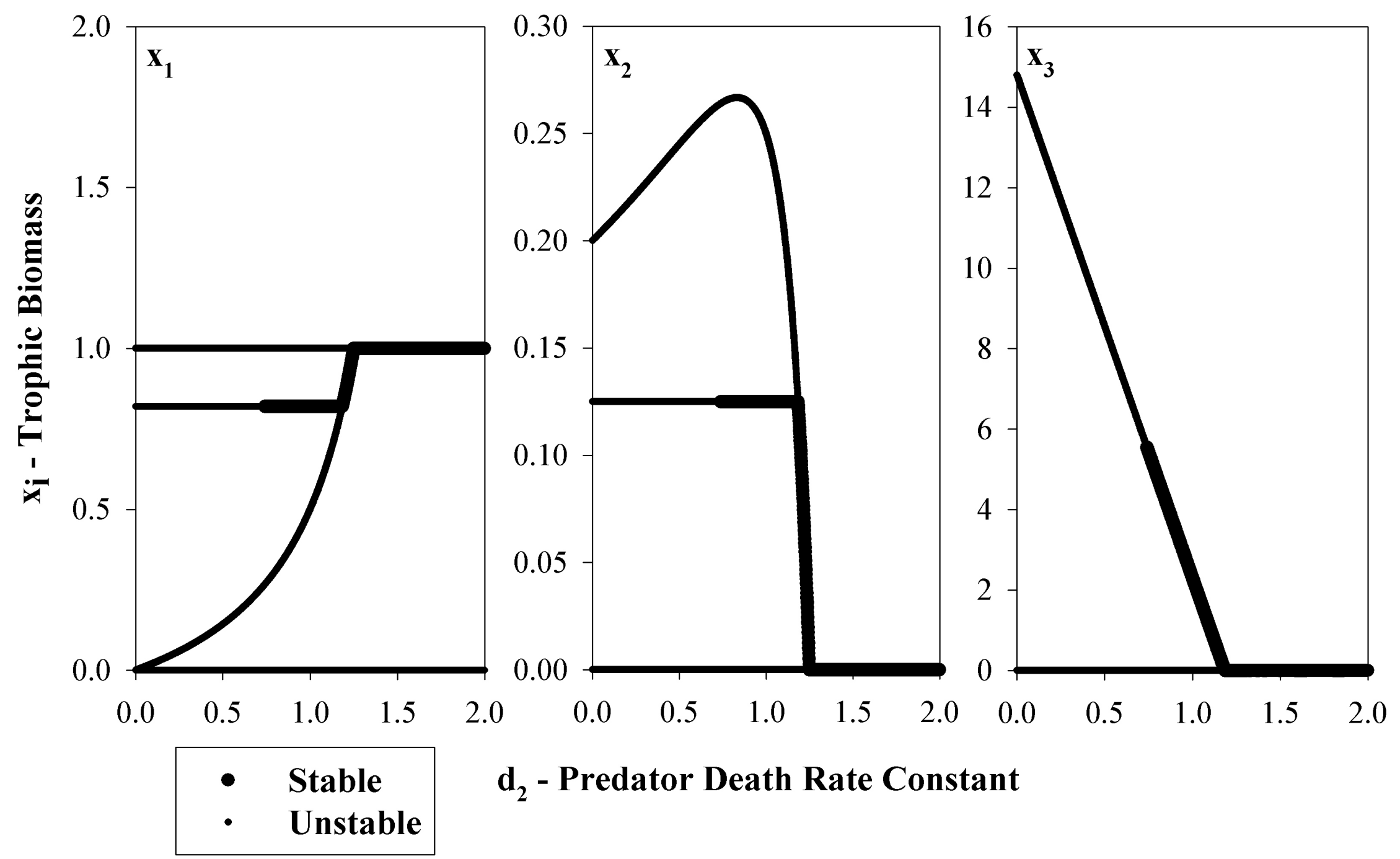

Figure 6. Solution branch diagrams illustrating the change in species biomass with the change in the predator death rate constant $d_{2}$ for the Rosenzweig-MacArthur model. From left to right: prey, predator, and superpredator biomasses. $\mathrm{K}=1.0$ and $\mathrm{r}=1.0 \mathrm{for}$ all three plots. 


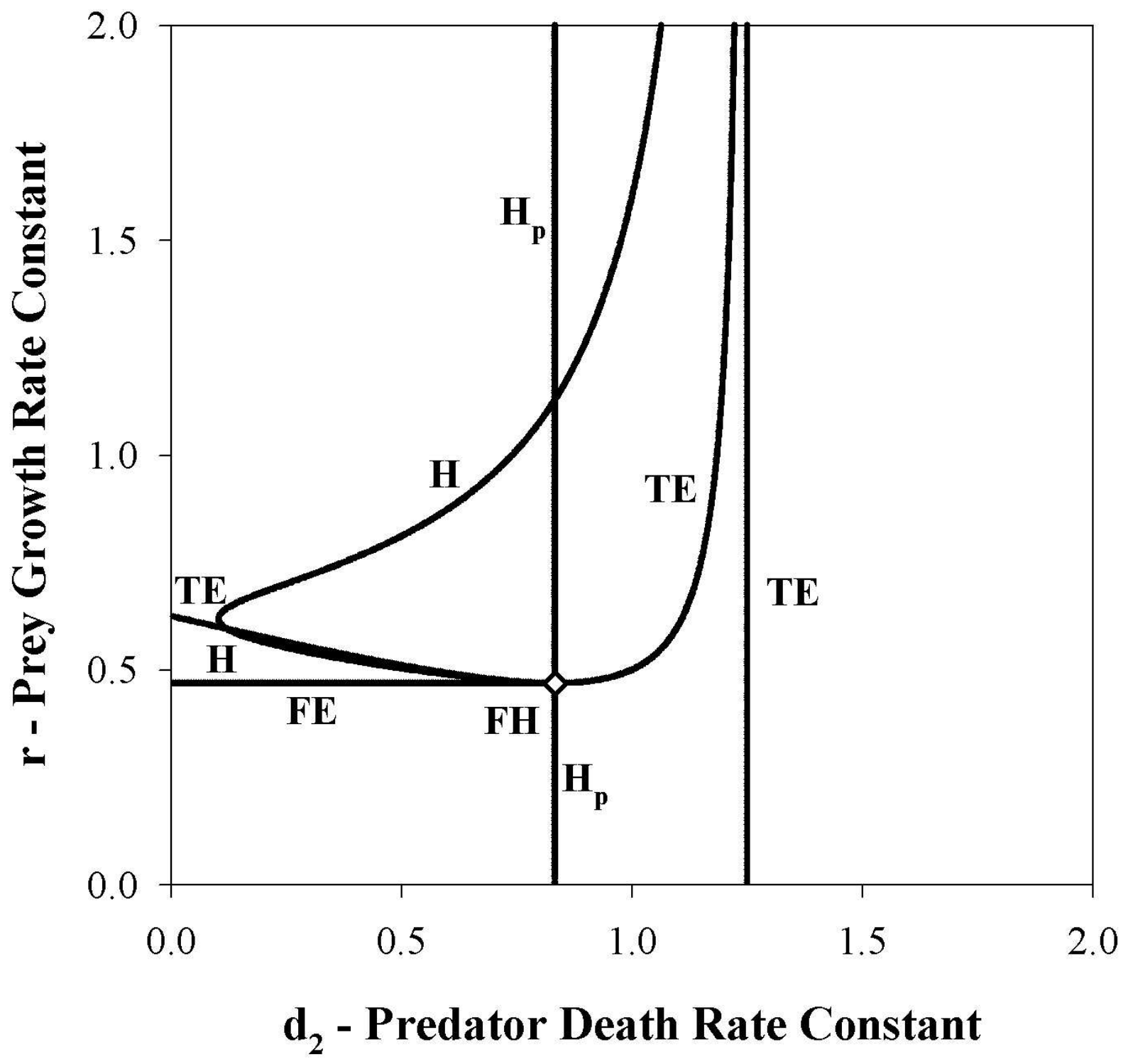

Figure 7. Bifurcation diagram of $d_{2}$ (predator death rate constant) vs. $r$ (prey growth rate constant) for the Rosenzweig-MacArthur model. $K=1.0$. 


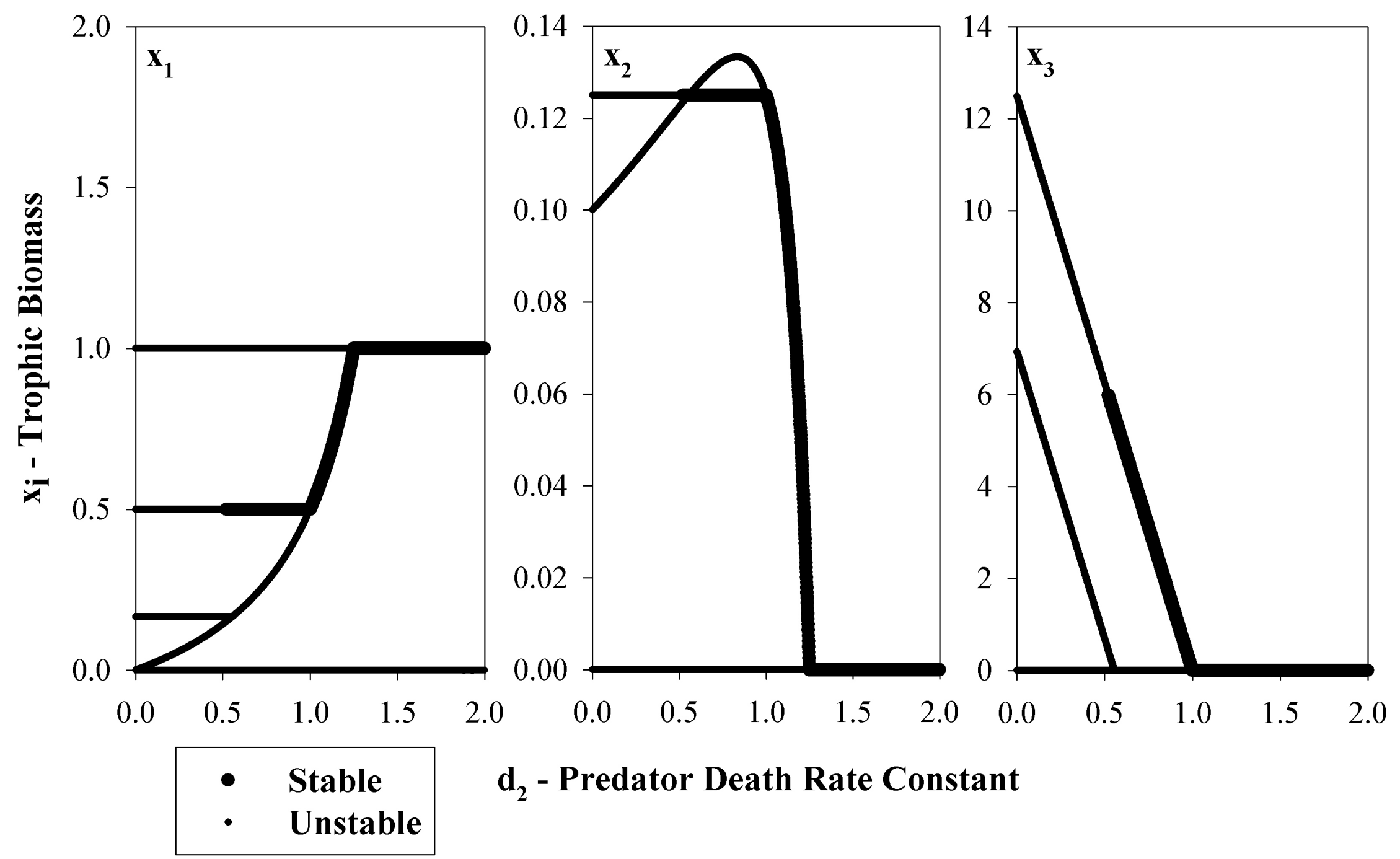

Figure 8. Solution branch diagrams illustrating the change in species biomass with the change in the predator death rate constant $d_{2}$ for the Rosenzweig-MacArthur model. From left to right: prey, predator, and superpredator biomasses. $K=1.0$ and $r=0.5$ for all three plots. 

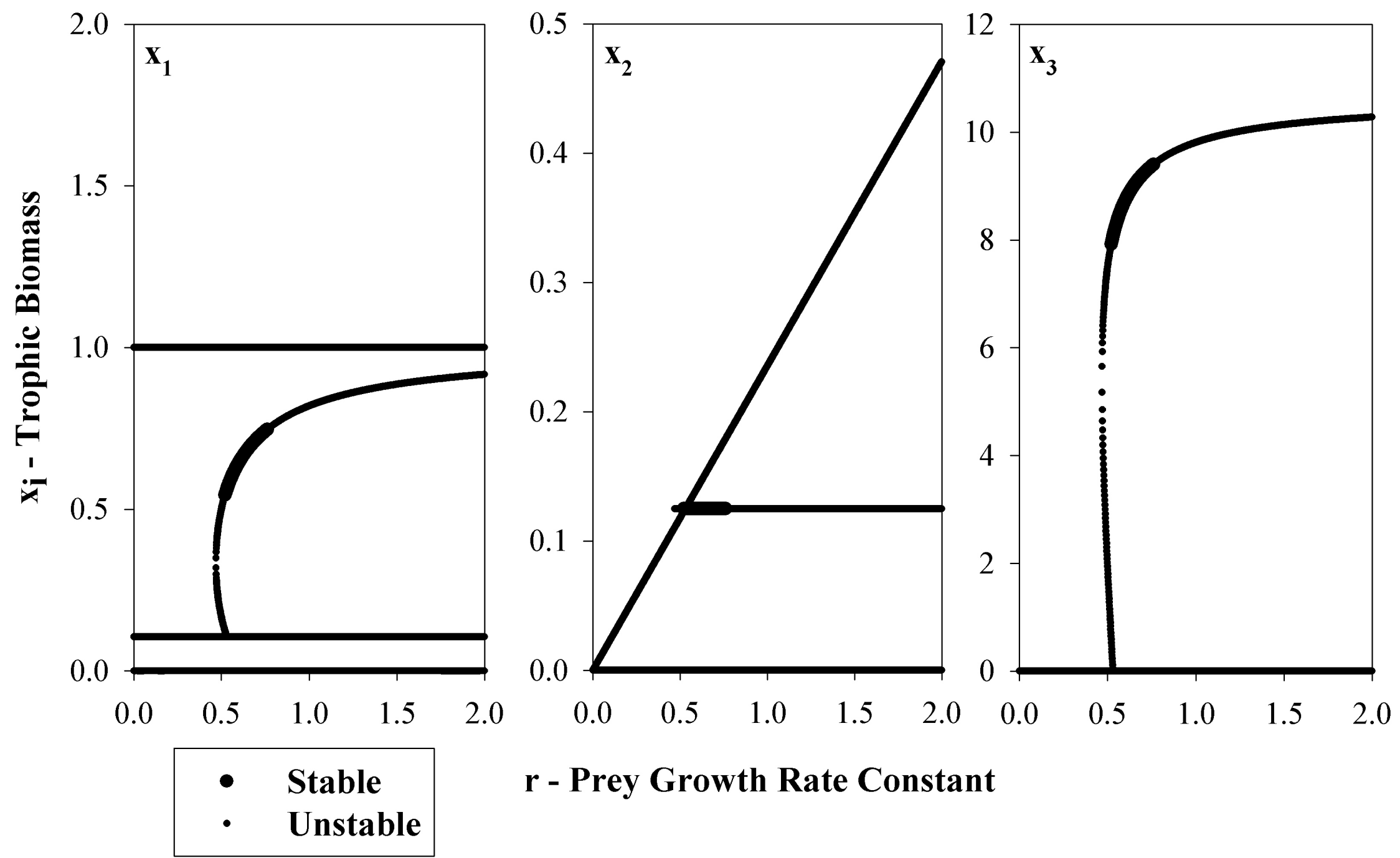

\section{r - Prey Growth Rate Constant}

Figure 9. Solution branch diagrams illustrating the change in species biomass with the change in the prey growth rate constant $r$ for the Rosenzweig-MacArthur model. From left to right: prey, predator, and superpredator biomasses. $K=1.0$ and $d_{2}=0.4$ in all three plots. 


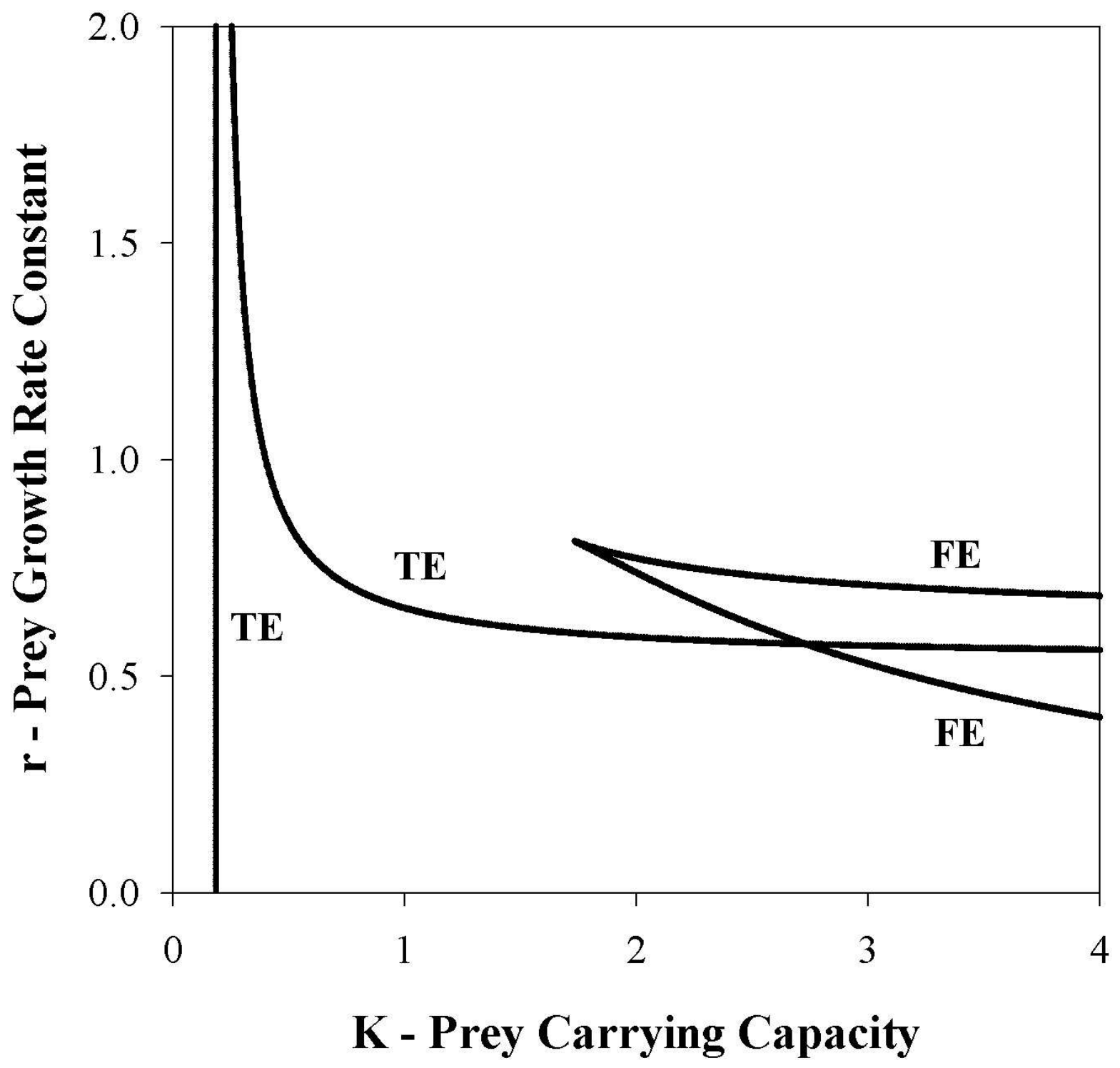

Figure 10. Bifurcation diagram of $K$ (prey carrying capacity) vs. $r$ (prey growth rate constant) for the tri-trophic, sigmoidal response model. 

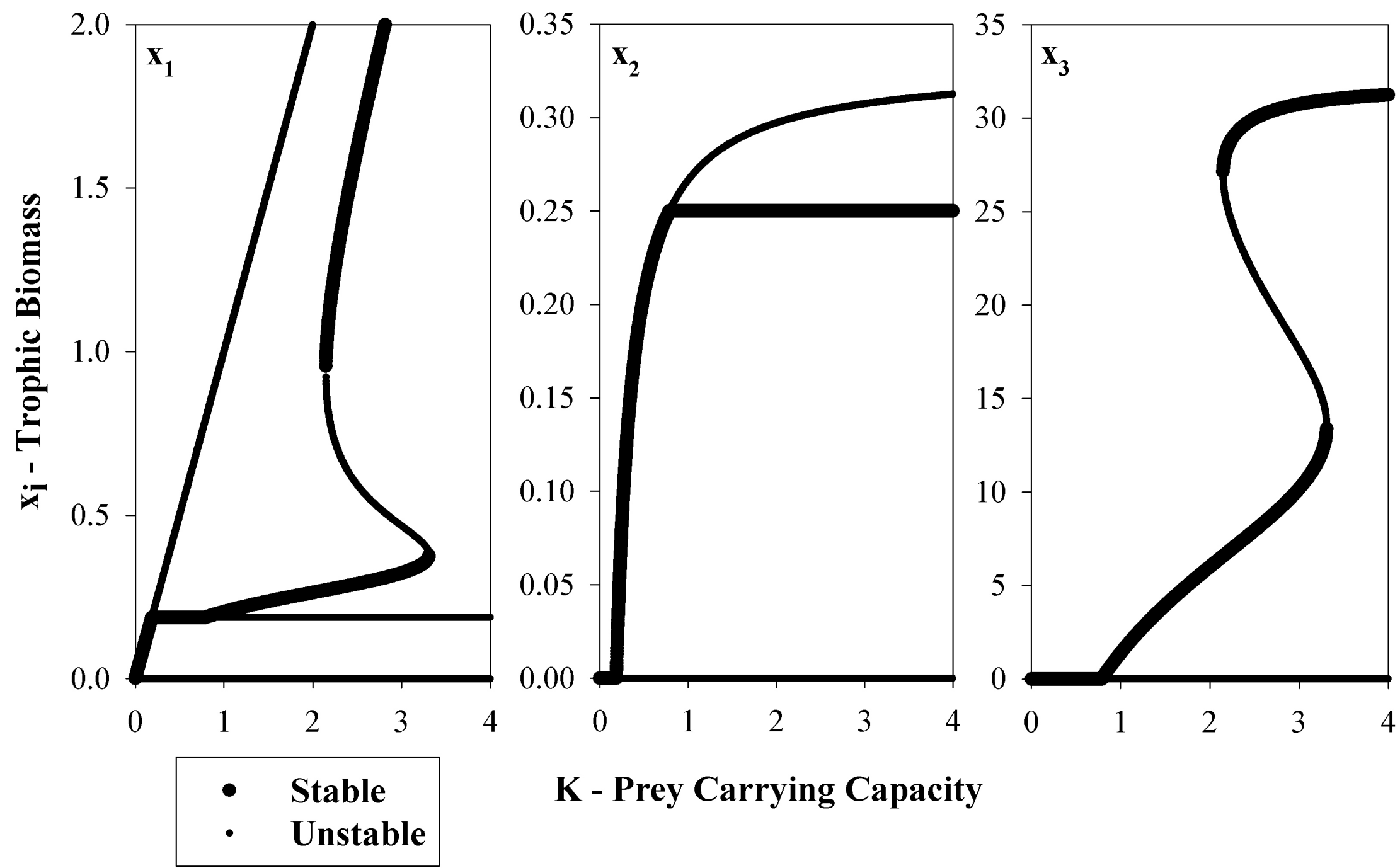

\section{K - Prey Carrying Capacity}

Figure 11. Solution branch diagrams illustrating the change in species biomass with the change in the prey carrying capacity $K$ for the tri-trophic, sigmoidal response model. From left to right: prey, predator, and superpredator biomasses. $r=0.7$ for all three plots. 


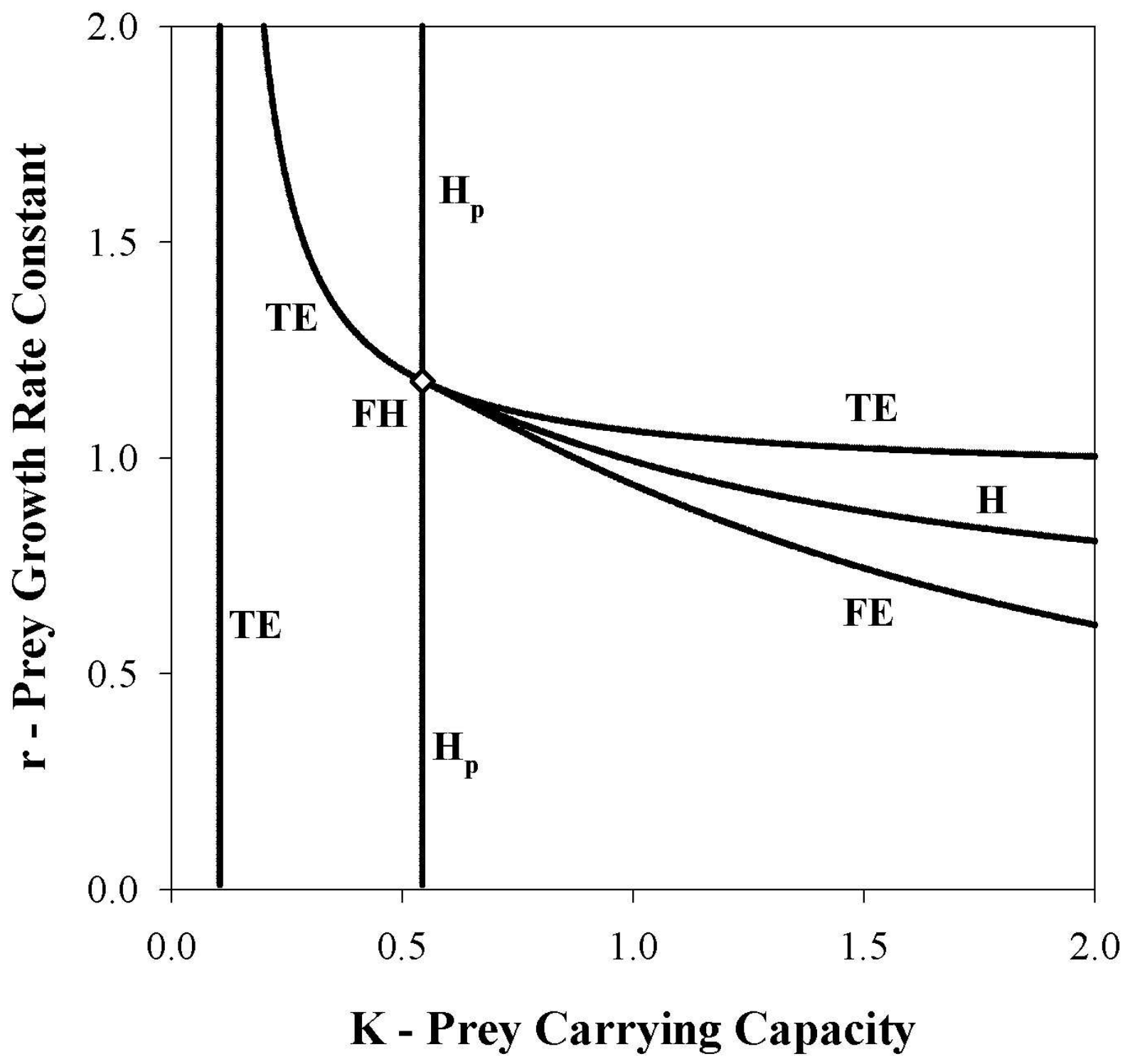

Figure 12. Bifurcation diagram of $K$ (prey carrying capacity) vs. $r$ (prey growth rate constant) for the tri-trophic model with a hyperbolic predator and a sigmoidal superpredator. 


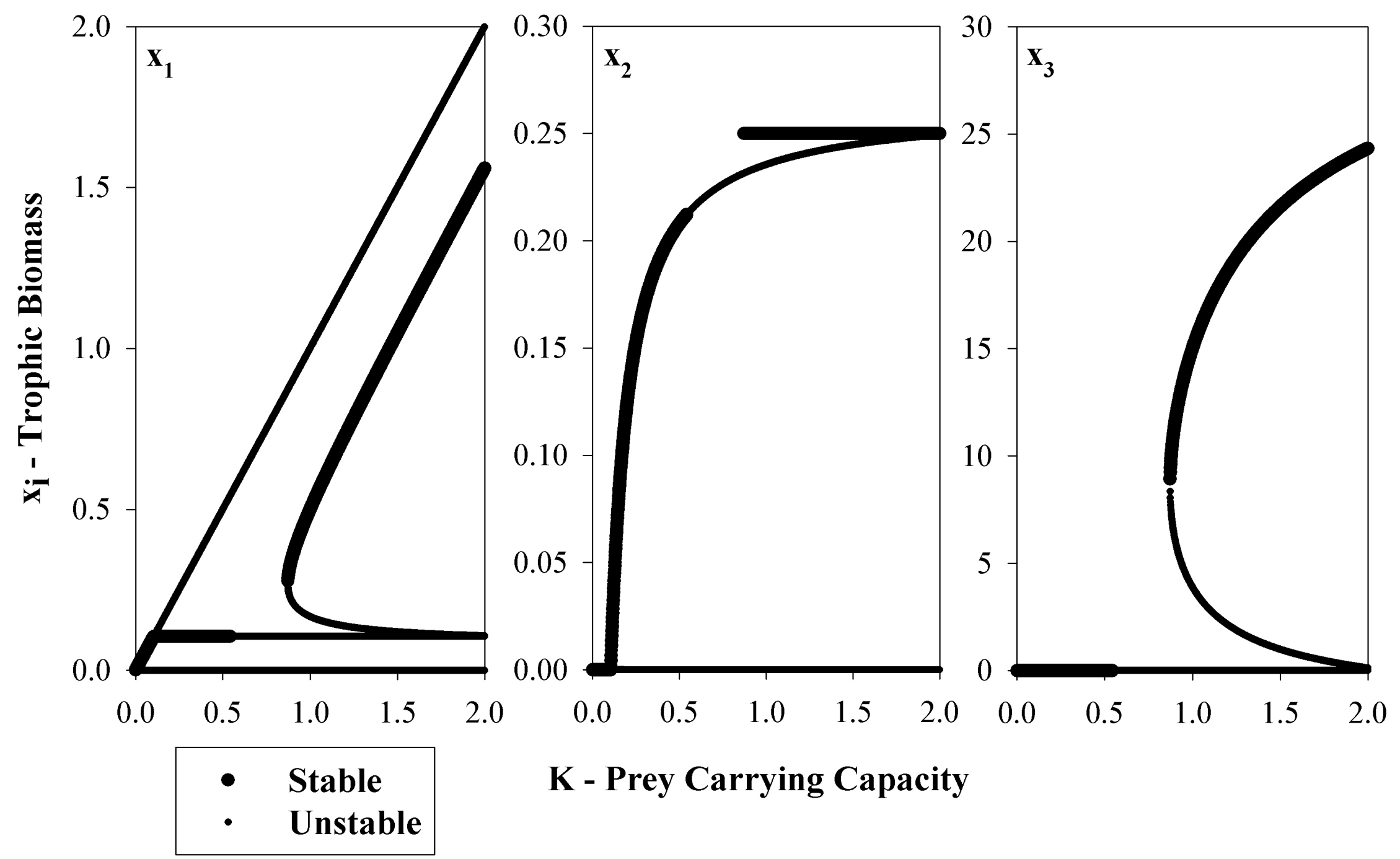

Figure 13. Solution branch diagrams illustrating the change in species biomass with the change in the prey carrying capacity $K$ for the tri-trophic model with a hyperbolic predator and a sigmoidal superpredator. From left to right: prey, predator, and superpredator biomasses. $r=1.0$ for all three plots. 